\title{
Selective Synthesis of $N$-Unsubstituted and $N$-Aryl Indoles by the Reaction of Arynes with Azirines
}

\author{
Manikandan Thangaraj, ${ }^{\dagger}{ }^{\star}$ Sachin Suresh Bhojgude,,${ }^{\dagger} \star$ Shailja Jain,,${ }^{\mathrm{t}}$ Rajesh G. \\ Gonnade $^{\S}$ and Akkattu T. Biju $*,+, \ddagger$ \\ ${ }^{\dagger}$ Organic Chemistry Division, ${ }^{E}$ Physical/Materials Chemistry Division ${ }^{\S}$ Centre for Materials \\ Characterization, CSIR-National Chemical Laboratory (CSIR-NCL), Dr. Homi Bhabha \\ Road, Pune - 411008, India. \\ ${ }^{\ddagger}$ Academy of Scientific and Innovative Research (AcSIR), New Delhi 110020, India \\ at.biju@ncl.res.in
}

\section{Supporting Information}

$\begin{array}{ll}\text { 1. X-Ray Data } & \text { S02 }\end{array}$

2. Computational Studies $\quad$ S04

3. ${ }^{1} \mathrm{H}$ and ${ }^{13} \mathrm{C}$ NMR Spectra of $N$-Unsubstituted Indoles $\quad \mathrm{S} 12$

4. ${ }^{1} \mathrm{H}$ and ${ }^{13} \mathrm{C}$ NMR Spectra of $N$-Aryl Indoles $\quad$ S29

5. ${ }^{1} \mathrm{H}$ and ${ }^{13} \mathrm{C}$ NMR Spectra of indolo[2,1-a]isoquinoline derivative S46 


\section{X-Ray Data}

X-ray intensity data measurements of compound were carried out on a CCD diffractometer with graphite-monochromatized $\left(\mathrm{MoK}_{\alpha}=0.71073 \AA\right)$ radiation. The X-ray generator was operated at $50 \mathrm{kV}$ and $30 \mathrm{~mA}$. A preliminary set of cell constants and an orientation matrix were calculated from three sets of 36 frames. Data were collected with $\omega$ scan width of $0.5^{\circ}$ at different settings of $\varphi$ and $2 \theta$ keeping the sample-to-detector distance fixed at $5.00 \mathrm{~cm}$. All the hydrogen atoms were placed in geometrically idealized position and constrained to ride on their parent atoms. An ORTEP III view of both compounds were drawn with $30 \%$ probability displacement ellipsoids and $\mathrm{H}$ atoms are shown as small spheres of arbitrary radii.

Crystal data of 3f: $\mathrm{C}_{22} \mathrm{H}_{13} \mathrm{~F}_{6} \mathrm{~N}, \mathrm{M}=405.33$, colorless plate, $0.43 \times 0.30 \times 0.26 \mathrm{~mm}^{3}$, triclinic, space group $P-1, a=7.8272(4) \AA, b=10.8142(5) \AA, c=11.7984(5) \AA, \alpha=$ $115.263(2)^{\circ}, \beta=91.231(3)^{\circ}, \gamma=91.976(3)^{\circ}, V=901.90(7) \AA^{3}, Z=2, T=150(2) \mathrm{K}, 2 \theta_{\max }=$ $50.00^{\circ}, D_{\text {calc }}\left(\mathrm{g} \mathrm{cm}^{-3}\right)=1.493, F(000)=412, \mu\left(\mathrm{mm}^{-1}\right)=0.131,13532$ reflections collected, 3175 unique reflections $\left(R_{\mathrm{int}}=0.0435\right), 2484$ observed $(I>2 \sigma(I))$ reflections, multi-scan absorption correction, $T_{\min }=0.946, T_{\max }=0.967,289$ refined parameters, 36 restraints, $S=$ 1.134, $R 1=0.0749, w R 2=0.1967$ (all data $R=0.0921, w R 2=0.2086$ ), maximum and minimum residual electron densities; $\Delta \rho_{\max }=0.526, \Delta \rho_{\min }=-0.414\left(\mathrm{e} \AA^{-3}\right)$.

Crystal data of $4 \mathbf{h}: \mathrm{C}_{26} \mathrm{H}_{17} \mathrm{Cl}_{2} \mathrm{~N}, \mathrm{M}=414.30$, colorless block, $0.49 \times 0.38 \times 0.27$ $\mathrm{mm}^{3}$, monoclinic, space group $P 2_{1} / c, a=11.346(3) \AA, b=20.720(6) \AA, c=8.864(3) \AA, \beta=$ $100.993(5)^{\circ}, V=2045.6(10) \AA^{3}, Z=4, T=130(2) \mathrm{K}, 2 \theta_{\max }=50.00^{\circ}, D_{\text {calc }}\left(\mathrm{g} \mathrm{cm}^{-3}\right)=1.345$, $F(000)=856, \mu\left(\mathrm{mm}^{-1}\right)=0.330,11241$ reflections collected, 3601 unique reflections $\left(R_{\text {int }}=0.0696\right), 2547$ observed $(I>2 \sigma(I))$ reflections, multi-scan absorption correction, $T_{\min }$ $=0.855, T_{\max }=0.916,262$ refined parameters, $S=1.069, R 1=0.0662, w R 2=0.1238$ (all data $R=0.1038, w R 2=0.1386$ ), maximum and minimum residual electron densities; $\Delta \rho_{\max }$ $=0.531, \Delta \rho_{\min }=-0.380\left(\mathrm{e} \AA^{-3}\right)$. 


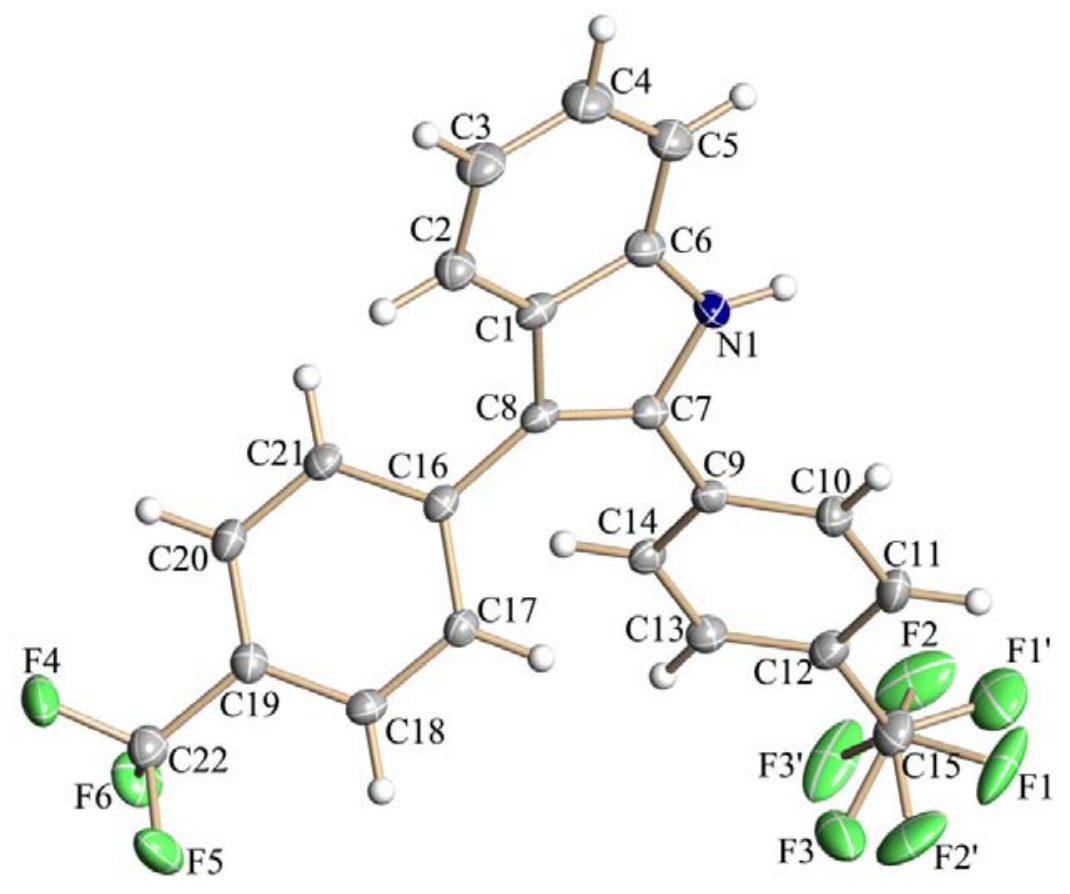

Fig S1: ORTEP Diagram of 3f. (thermal ellipsoids are shown with 30\% probability).

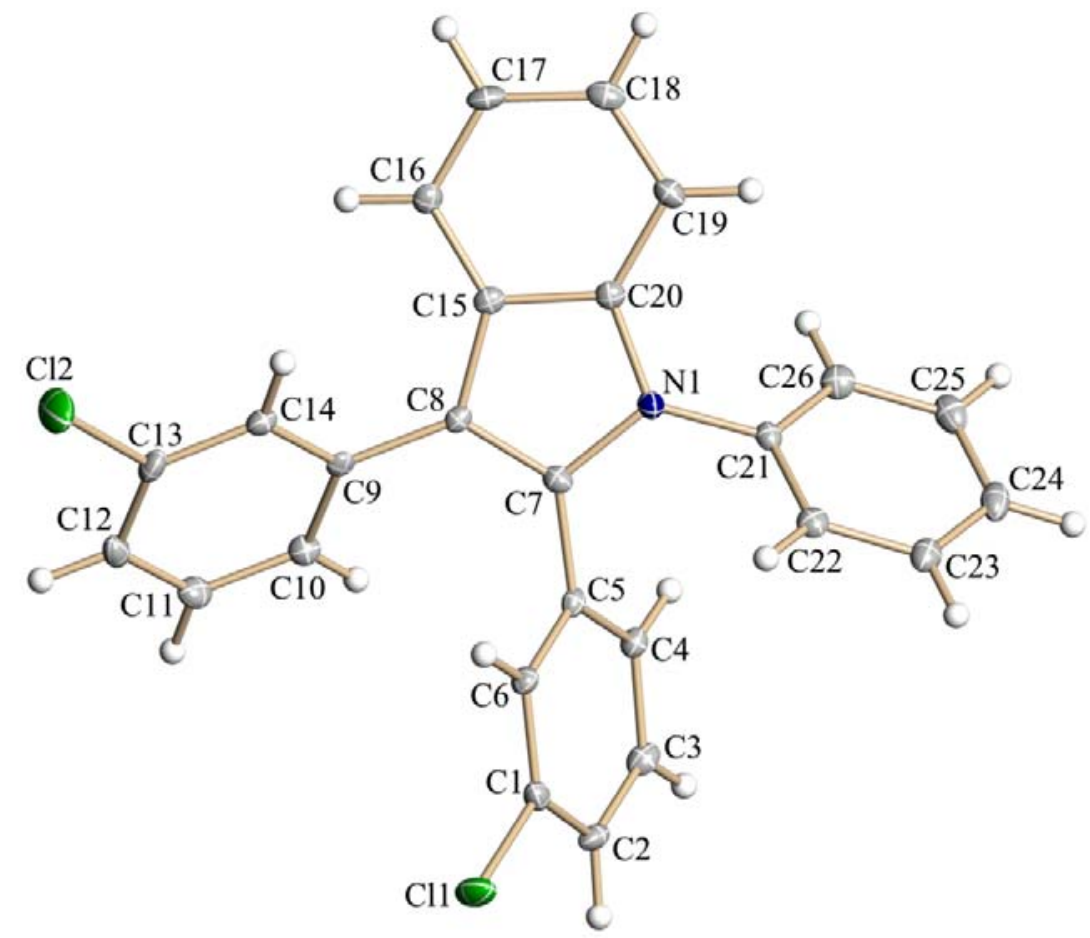

Fig S2: ORTEP Diagram of 4 h. (thermal ellipsoids are shown with 30\% probability). 


\section{Computational Studies}

The geometry optimizations were conducted employing density functional theory (DFT). ${ }^{1,2}$ The triple- $\zeta$ basis set augmented by a polarization function was used for all the atoms. The resolution of identity $(\mathrm{RI})^{3}$ along with the multipole accelerated resolution of identity (marij) ${ }^{4}$ approximations were employed for an accurate and efficient treatment of the electronic Coulomb term. Solvent effects were accounted for as follows: we have done full geometry optimizations of all intermediates and transition states calculations using the COSMO model. The solvent used in this study is THF $(\varepsilon=7.58)$. The contributions of internal energy and entropy were obtained from frequency calculations done on the DFT structures: thus, the energies reported in the figures are the $\Delta \mathrm{G}$ values. To gain insight into the mechanism, we performed quantum chemical calculations by using density functional theory (DFT), employing the TZVP/PBE approach.

Herein we proposed two mechanisms based on the temperature effect. In the first pathway, at $60{ }^{\circ} \mathrm{C}$ formation of $\mathbf{3}$ is preferred, and in second pathway, at $-10{ }^{\circ} \mathrm{C}$, the formation of $\mathbf{4}$ is preferred. In both the pathways, common intermediate $\mathbf{A}$ is forming. The results are summarized in terms of a free energy values.

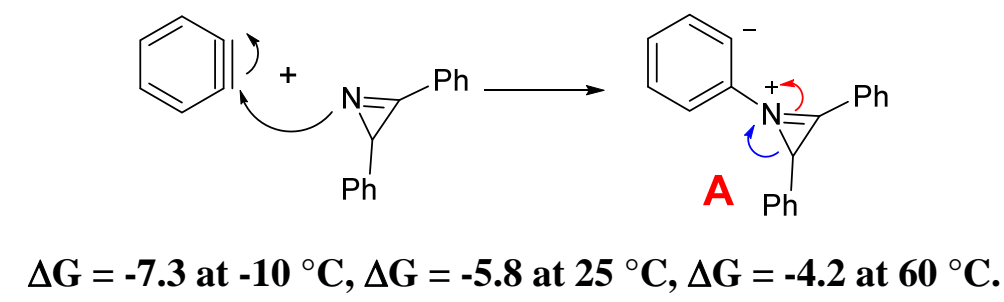

Fig S3: Formation of intermediate A.

Formation of intermediate $\mathbf{A}$ was found to $-5.8 \mathrm{kcal} / \mathrm{mol}$ at room temperature, this is an indication that it is common intermediate for both the pathways ( $a$ and b). These results are confirming the formation of intermediate $\mathbf{A}$ in the reaction course.

\footnotetext{
${ }^{1}$ Ahlrichs, R.; Bar, M.; Haser, M.; Horn, H.; Kolmel, C Chem. Phy. Lett. 1989, 162, 165.

${ }^{2}$ Perdew, J. P.; Burke, K.; Ernzerhof, M. Phy. Rev. Lett. 1996, 77, 3865.

${ }^{3}$ Eichkorn, K.; Treutler, O.; Öhm, H.; Häser, M.; Ahlrichs, R. Chem. Phy. Lett. 1995, 240, 283.

${ }^{4}$ Sierka, M.; Hogekamp, A.; Ahlrichs, R. J. Chem. Phys. 2003, 118, 91.
} 


\section{$\Delta \mathrm{G}$ at $60^{\circ} \mathrm{C}$ in $\mathrm{Kcal} / \mathrm{mol}$}

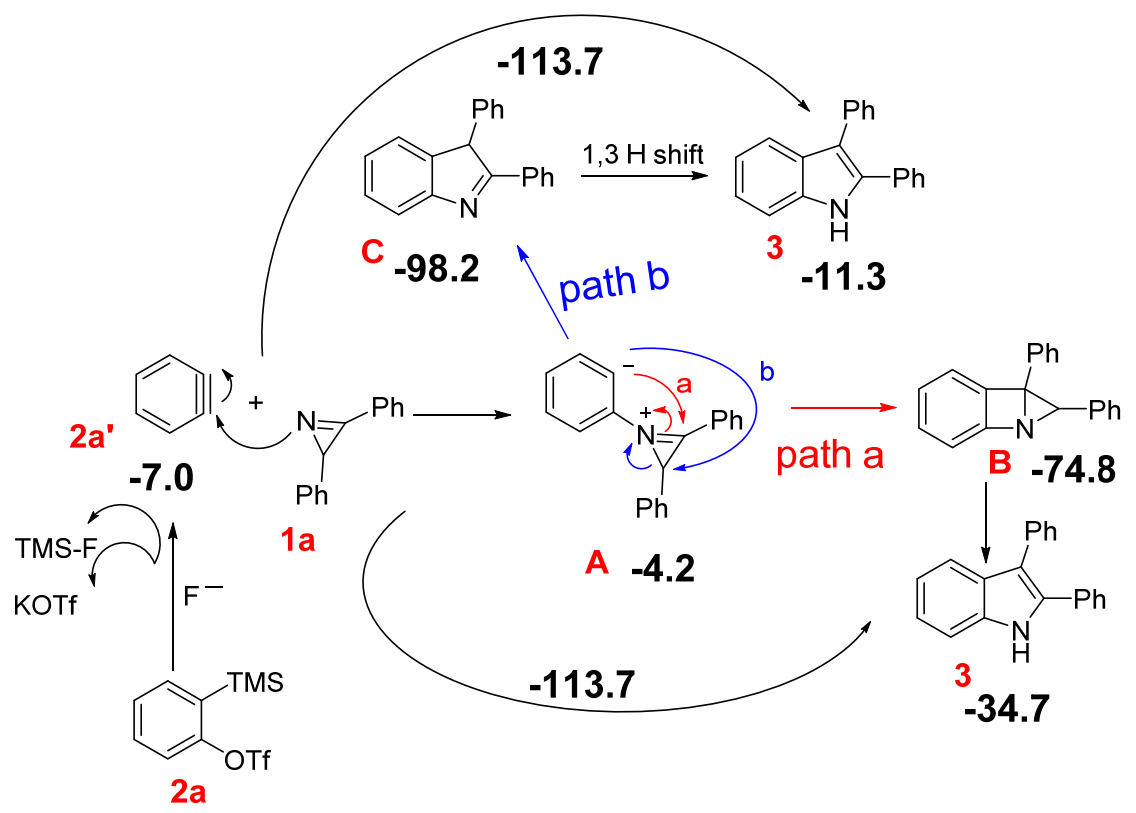

Fig S4: Formation of product 3.

The formation of Product 3 proceed via both the pathways ( $a$ and $b$ ), which is -113.7 $\mathrm{kcal} / \mathrm{mol}$ stabilized, with respect to azirine $1 \mathrm{a}$ and benzyne $2 \mathrm{a}^{\prime}$ at $60^{\circ} \mathrm{C}$.

\section{$\Delta \mathrm{G}$ at $-10^{\circ} \mathrm{C}$ in $\mathrm{Kcal} / \mathrm{mol}$}

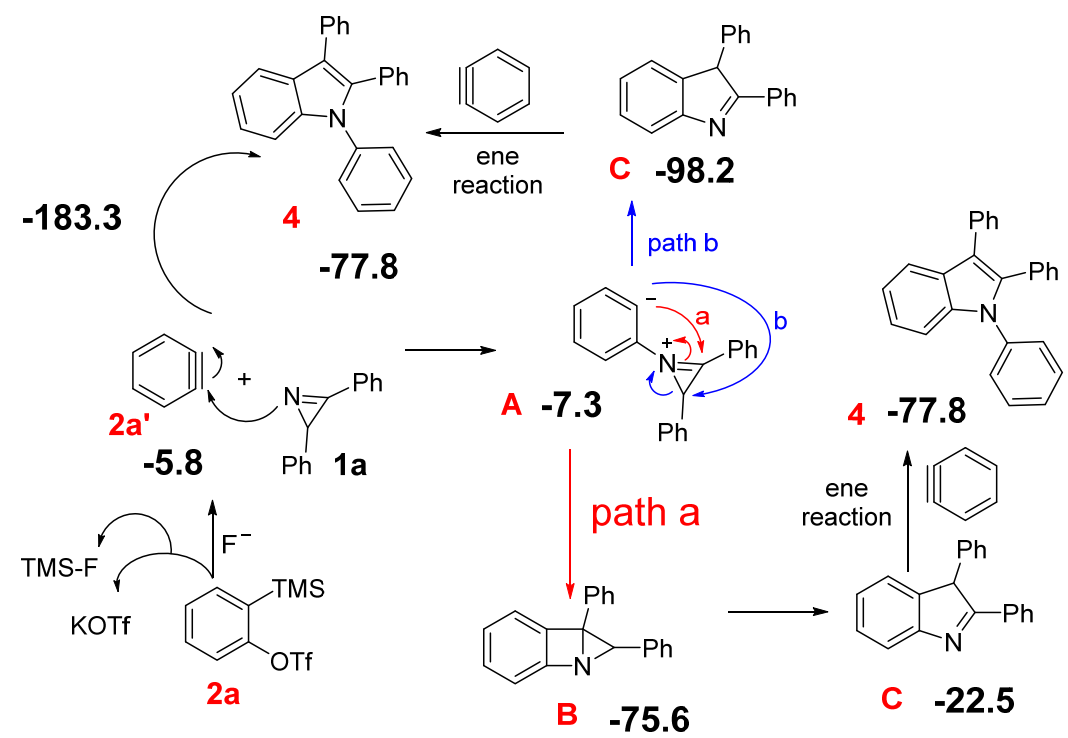

Fig S5: Formation of product 4

The formation of Product $\mathbf{4}$ proceed via both the pathways ( $a$ and $b$ ) which is -183 $\mathrm{kcal} / \mathrm{mol}$ stabilized with respect to azirine $\mathbf{1 a}$ and benzyne $2 \mathbf{a}^{\prime}$ at $-10^{\circ} \mathrm{C}$. 
II. The optimized geometries of the structures reported in the manuscript (the atomic symbol followed by the three Cartesian coordinates, in Å).

\begin{tabular}{|c|c|c|c|c|c|c|c|}
\hline \multicolumn{8}{|c|}{$2 \mathrm{a}$} \\
\hline \multicolumn{4}{|c|}{31} & \multicolumn{4}{|c|}{ K-OTf } \\
\hline $\mathrm{C}$ & 2.158 & 0.137 & 0.046 & 9 & & & \\
\hline $\mathrm{C}$ & 0.949 & 0.005 & -0.665 & $\mathrm{C}$ & 3.204 & 0.064 & 2.469 \\
\hline $\mathrm{C}$ & -0.203 & -0.118 & 0.120 & $\mathrm{~F}$ & 3.690 & -1.153 & 2.790 \\
\hline $\mathrm{C}$ & -0.194 & -0.137 & 1.514 & $\mathrm{~S}$ & 1.372 & 0.220 & 2.997 \\
\hline $\mathrm{C}$ & 1.025 & 0.018 & 2.178 & $\mathrm{O}$ & 1.400 & 0.020 & 4.478 \\
\hline $\mathrm{C}$ & 2.204 & 0.156 & 1.443 & $\mathrm{O}$ & 0.683 & -0.861 & 2.196 \\
\hline $\mathrm{Si}$ & 0.961 & -0.009 & -2.589 & $\mathrm{~F}$ & 3.322 & 0.230 & 1.130 \\
\hline $\mathrm{C}$ & 0.138 & 1.571 & -3.239 & $\mathrm{~F} ?$ & 3.957 & 1.001 & 3.081 \\
\hline $\mathrm{O}$ & -1.421 & -0.313 & -0.603 & $\mathrm{~K}$ & -0.815 & 0.829 & 0.473 \\
\hline S & -2.848 & 0.321 & -0.013 & $\mathrm{O}$ & 0.988 & 1.603 & 2.523 \\
\hline $\mathrm{O}$ & -3.460 & -0.606 & 0.959 & & & & \\
\hline $\mathrm{C}$ & 2.771 & -0.038 & -3.153 & & & & \\
\hline $\mathrm{C}$ & 0.093 & -1.566 & -3.241 & $\mathrm{KF}$ & & & \\
\hline $\mathrm{C}$ & -3.798 & 0.109 & -1.684 & 2 & & & \\
\hline $\mathrm{F}$ & -3.654 & -1.134 & -2.144 & $\mathrm{~K}$ & 0.000 & 0.000 & -0.060 \\
\hline $\mathrm{F}$ & -5.085 & 0.356 & -1.422 & $\mathrm{~F}$ & 0.000 & 0.000 & 2.382 \\
\hline $\mathrm{F}$ & -3.337 & 0.988 & -2.577 & & & & \\
\hline $\mathrm{H}$ & -1.112 & -0.279 & 2.087 & & & & \\
\hline $\mathrm{H}$ & 1.044 & 0.017 & 3.269 & & & & \\
\hline $\mathrm{H}$ & 3.160 & 0.275 & 1.957 & & & & \\
\hline $\mathrm{H}$ & 3.093 & 0.233 & -0.510 & & & & \\
\hline $\mathrm{H}$ & 0.147 & 1.571 & -4.340 & & & & \\
\hline $\mathrm{H}$ & -0.904 & 1.663 & -2.908 & & & & \\
\hline $\mathrm{H}$ & 0.687 & 2.459 & -2.892 & & & & \\
\hline $\mathrm{H}$ & 0.154 & -1.591 & -4.340 & & & & \\
\hline $\mathrm{H}$ & 0.580 & -2.473 & -2.852 & & & & \\
\hline $\mathrm{H}$ & -0.966 & -1.599 & -2.954 & & & & \\
\hline $\mathrm{H}$ & 2.800 & -0.072 & -4.254 & & & & \\
\hline $\mathrm{H}$ & 3.320 & 0.859 & -2.833 & & & & \\
\hline $\mathrm{H}$ & 3.303 & -0.925 & -2.777 & & & & \\
\hline $\mathrm{O}$ & -2.710 & 1.763 & 0.274 & & & & \\
\hline \multicolumn{8}{|c|}{ TMS-F } \\
\hline \multicolumn{8}{|c|}{14} \\
\hline $\mathrm{Si}$ & 0.044 & -0.076 & 0.031 & & & & \\
\hline $\mathrm{C}$ & -0.009 & 0.018 & 1.909 & & & & \\
\hline $\mathrm{C}$ & 1.796 & 0.018 & -0.646 & & & & \\
\hline $\mathrm{C}$ & -0.914 & -1.547 & -0.646 & & & & \\
\hline $\mathrm{F}$ & -0.749 & 1.294 & -0.527 & & & & \\
\hline $\mathrm{H}$ & -0.923 & -1.536 & -1.746 & & & & \\
\hline $\mathrm{H}$ & -0.449 & -2.489 & -0.318 & & & & \\
\hline $\mathrm{H}$ & -1.954 & -1.537 & -0.289 & & & & \\
\hline $\mathrm{H}$ & 1.794 & 0.031 & -1.746 & & & & \\
\hline $\mathrm{H}$ & 2.308 & 0.923 & -0.289 & & & & \\
\hline
\end{tabular}



$\begin{array}{llll}\mathrm{H} & 2.379 & -0.855 & -0.317\end{array}$
$\begin{array}{llll}\mathrm{H} & -1.048 & 0.032 & 2.272\end{array}$
$\begin{array}{llll}\mathrm{H} & 0.493 & -0.855 & 2.350\end{array}$
$\begin{array}{llll}\mathrm{H} & 0.497 & 0.924 & 2.272\end{array}$

\begin{tabular}{|c|c|c|c|c|}
\hline \multicolumn{5}{|c|}{$2 a^{\prime}$} \\
\hline & & & & \\
\hline $\mathrm{C}$ & 0.093916 & 16 & -0.000009 & 0.071953 \\
\hline $\mathrm{C}$ & 0.093355 & 55 & 0.000008 & 1.328284 \\
\hline $\mathrm{C}$ & \multicolumn{2}{|c|}{1.194692} & 0.000039 & 2.168561 \\
\hline $\mathrm{C}$ & \multicolumn{2}{|c|}{2.388755} & 0.000000 & 1.402884 \\
\hline $\mathrm{C}$ & 2.388641 & 41 & -0.000007 & -0.002963 \\
\hline $\mathrm{C}$ & \multicolumn{2}{|c|}{1.194742} & -0.000038 & -0.768615 \\
\hline $\mathrm{H}$ & \multicolumn{2}{|c|}{1.196857} & -0.000020 & 3.258545 \\
\hline $\mathrm{H}$ & \multicolumn{2}{|c|}{3.343790} & -0.000004 & 1.934705 \\
\hline $\mathrm{H}$ & 3.343800 & 300 & 0.000007 & -0.534735 \\
\hline $\mathrm{H}$ & \multicolumn{2}{|c|}{1.196886} & 0.000024 & -1.858618 \\
\hline \multicolumn{5}{|c|}{$1 \mathrm{a}$} \\
\hline \multicolumn{5}{|l|}{26} \\
\hline $\mathrm{N}$ & -0.811 & -0.942 & -0.194 & \\
\hline $\mathrm{C}$ & -0.013 & -0.208 & 0.940 & \\
\hline $\mathrm{C}$ & 0.322 & -0.432 & -0.460 & \\
\hline $\mathrm{C}$ & -0.519 & 1.099 & 1.427 & \\
\hline $\mathrm{C}$ & 1.229 & -0.176 & -1.555 & \\
\hline $\mathrm{C}$ & -0.427 & 1.414 & 2.794 & \\
\hline $\mathrm{C}$ & -0.883 & 2.644 & 3.276 & \\
\hline $\mathrm{C}$ & -1.444 & 3.578 & 2.399 & \\
\hline $\mathrm{C}$ & -1.547 & 3.271 & 1.036 & \\
\hline $\mathrm{C}$ & -1.089 & 2.043 & 0.553 & \\
\hline $\mathrm{H}$ & 0.005 & 0.685 & 3.483 & \\
\hline $\mathrm{H}$ & -0.805 & 2.875 & 4.340 & \\
\hline $\mathrm{H}$ & -1.800 & 4.538 & 2.776 & \\
\hline $\mathrm{H}$ & -1.986 & 3.995 & 0.347 & \\
\hline $\mathrm{H}$ & -1.177 & 1.815 & -0.511 & \\
\hline $\mathrm{C}$ & 2.449 & 0.477 & -1.304 & \\
\hline $\mathrm{C}$ & 3.325 & 0.740 & -2.357 & \\
\hline $\mathrm{C}$ & 2.983 & 0.364 & -3.661 & \\
\hline $\mathrm{C}$ & 1.765 & -0.282 & -3.914 & \\
\hline $\mathrm{C}$ & 0.888 & -0.557 & -2.868 & \\
\hline $\mathrm{H}$ & 2.698 & 0.775 & -0.283 & \\
\hline $\mathrm{H}$ & 4.273 & 1.245 & -2.161 & \\
\hline $\mathrm{H}$ & 3.666 & 0.573 & -4.488 & \\
\hline $\mathrm{H}$ & 1.500 & -0.571 & -4.933 & \\
\hline $\mathrm{H}$ & -0.062 & -1.060 & -3.057 & \\
\hline $\mathrm{H}$ & 0.408 & -0.871 & 1.702 & \\
\hline
\end{tabular}




\begin{tabular}{|c|c|c|c|}
\hline \multicolumn{4}{|l|}{ A } \\
\hline \multicolumn{4}{|l|}{36} \\
\hline $\mathrm{C}$ & -0.615431 & -0.646406 & -2.433582 \\
\hline $\mathrm{C}$ & -0.935756 & -1.602415 & -1.445217 \\
\hline $\mathrm{C}$ & -1.952298 & -2.584244 & -1.486449 \\
\hline $\mathrm{C}$ & -2.739802 & -2.642866 & -2.629999 \\
\hline $\mathrm{C}$ & -2.491478 & -1.731746 & -3.672389 \\
\hline $\mathrm{C}$ & -1.469065 & -0.775884 & -3.557367 \\
\hline $\mathrm{N}$ & -0.154407 & -1.597061 & -0.269672 \\
\hline $\mathrm{C}$ & 0.791326 & -1.091966 & 0.434647 \\
\hline $\mathrm{C}$ & 1.802747 & -0.093319 & 0.451327 \\
\hline $\mathrm{C}$ & 1.968497 & 0.759798 & -0.665814 \\
\hline $\mathrm{C}$ & 2.971871 & 1.723785 & -0.633997 \\
\hline $\mathrm{C}$ & 3.804916 & 1.842141 & 0.487858 \\
\hline $\mathrm{C}$ & 3.636659 & 0.997376 & 1.594647 \\
\hline $\mathrm{C}$ & 2.637264 & 0.029213 & 1.585131 \\
\hline $\mathrm{C}$ & 0.024244 & -2.182777 & 1.104722 \\
\hline $\mathrm{C}$ & -1.010954 & -1.910135 & 2.140849 \\
\hline $\mathrm{C}$ & -1.589124 & -0.638083 & 2.285545 \\
\hline $\mathrm{C}$ & -2.567138 & -0.418359 & 3.256650 \\
\hline $\mathrm{C}$ & -2.974823 & -1.463793 & 4.094893 \\
\hline $\mathrm{C}$ & -2.401366 & -2.731678 & 3.955948 \\
\hline $\mathrm{C}$ & -1.424565 & -2.954598 & 2.981048 \\
\hline $\mathrm{H}$ & 0.462557 & -3.186871 & 1.112918 \\
\hline $\mathrm{H}$ & -3.013148 & 0.572462 & 3.360997 \\
\hline $\mathrm{H}$ & -1.280943 & 0.182017 & 1.632244 \\
\hline $\mathrm{H}$ & -0.980212 & -3.946569 & 2.867936 \\
\hline $\mathrm{H}$ & -2.718077 & -3.551043 & 4.603950 \\
\hline $\mathrm{H}$ & -3.740362 & -1.289294 & 4.853238 \\
\hline $\mathrm{H}$ & 1.286188 & 0.622394 & -1.519805 \\
\hline $\mathrm{H}$ & 3.110829 & 2.389413 & -1.488016 \\
\hline $\mathrm{H}$ & 4.591995 & 2.598769 & 0.501868 \\
\hline $\mathrm{H}$ & 4.289199 & 1.098413 & 2.463348 \\
\hline $\mathrm{H}$ & 2.493526 & -0.634687 & 2.439806 \\
\hline $\mathrm{H}$ & -2.116949 & -3.269361 & -0.649555 \\
\hline $\mathrm{H}$ & -3.537684 & -3.383002 & -2.714044 \\
\hline $\mathrm{H}$ & -3.107176 & -1.773948 & -4.576695 \\
\hline $\mathrm{H}$ & -1.335913 & -0.092469 & -4.407106 \\
\hline \multicolumn{4}{|c|}{$\mathrm{C}$} \\
\hline \multicolumn{4}{|l|}{36} \\
\hline $\mathrm{N}$ & 0.155641 & 0.473413 & -0.129065 \\
\hline $\mathrm{C}$ & 0.228465 & 0.130002 & 1.237086 \\
\hline $\mathrm{C}$ & 1.389280 & -0.614890 & 1.531996 \\
\hline $\mathrm{C}$ & 2.150289 & -0.772723 & 0.235461 \\
\hline $\mathrm{C}$ & 1.218418 & -0.011730 & -0.718546 \\
\hline $\mathrm{C}$ & 1.461010 & 0.143771 & -2.154602 \\
\hline $\mathrm{C}$ & 3.582100 & -0.270581 & 0.297633 \\
\hline $\mathrm{C}$ & 1.651769 & -1.042680 & 2.826506 \\
\hline $\mathrm{C}$ & -0.698729 & 0.450433 & 2.231825 \\
\hline $\mathrm{C}$ & 2.535697 & -0.512888 & -2.787379 \\
\hline
\end{tabular}




\begin{tabular}{|c|c|c|c|}
\hline & & & \\
\hline & 1.895014 & 0.435536 & \\
\hline & 0.823980 & & \\
\hline & 0.60 & & \\
\hline & & & \\
\hline & 3.584264 & -0.885361 & \\
\hline & & & \\
\hline & & & \\
\hline & -0.222782 & & \\
\hline & & -1.1 & \\
\hline & & -0.7 & \\
\hline & & & \\
\hline$y$ & & & \\
\hline & & & \\
\hline & 4.4 & -2.2 & \\
\hline & & -1. & \\
\hline I & 7.2 & & \\
\hline & & & \\
\hline & 3.0 & & \\
\hline C & & -0.7 & \\
\hline$y$ & -0.4 & & \\
\hline [ & & & \\
\hline & & & \\
\hline I & -1.1 & & \\
\hline$H$ & & & \\
\hline & & & \\
\hline \\
\hline \\
\hline & & & \\
\hline $\mathrm{C}$ & -0.0 & & \\
\hline $\mathrm{C}$ & & -0.0 & \\
\hline $\mathrm{C}$ & & & \\
\hline$r$ & & -0.0 & \\
\hline & & -0.0 & \\
\hline $\mathrm{C}$ & & & \\
\hline r & & & \\
\hline & -1.2 & -0.2 & \\
\hline & -1.2 & & \\
\hline & $-2.4 c$ & & \\
\hline C & -3.6 & -0.5 & \\
\hline & -3.65 & & \\
\hline $\mathrm{C}$ & $-2.4 \varepsilon$ & & \\
\hline $\mathrm{H}$ & -0.3 & & \\
\hline & -2.45 & & \\
\hline $\mathrm{H}$ & -4.557805 & & \\
\hline $\mathrm{H}$ & -4.566515 & & \\
\hline & -2.49 & & \\
\hline & 2.939788 & -0.64 & \\
\hline$r$ & 3.463947 & -0.519187 & \\
\hline $\mathrm{C}$ & 2.875150 & 0.355979 & 5.79997 \\
\hline
\end{tabular}




\begin{tabular}{|c|c|c|c|}
\hline $\mathrm{C}$ & 1.760645 & 1.110448 & 5.414276 \\
\hline $\mathrm{C}$ & 1.241822 & 0.991419 & 4.124104 \\
\hline $\mathrm{H}$ & 3.391714 & -1.348734 & 2.889704 \\
\hline $\mathrm{H}$ & 4.332764 & -1.114320 & 5.171093 \\
\hline $\mathrm{H}$ & 3.283169 & 0.452146 & 6.808222 \\
\hline $\mathrm{H}$ & 1.298333 & 1.803715 & 6.120549 \\
\hline $\mathrm{H}$ & 0.381233 & 1.593712 & 3.826295 \\
\hline $\mathrm{H}$ & -0.998003 & -0.311456 & -0.451022 \\
\hline $\mathrm{C}$ & 3.822467 & 0.174144 & -1.016175 \\
\hline $\mathrm{C}$ & 2.888083 & 0.071322 & -2.071465 \\
\hline $\mathrm{H}$ & 0.795647 & -0.102500 & -2.630068 \\
\hline $\mathrm{H}$ & 3.240967 & 0.083781 & -3.104415 \\
\hline $\mathrm{H}$ & 4.884153 & 0.271349 & -1.251891 \\
\hline $\mathrm{H}$ & 4.145886 & 0.240503 & 1.120674 \\
\hline \multicolumn{4}{|l|}{ B } \\
\hline \multicolumn{4}{|c|}{36} \\
\hline $\mathrm{C}$ & -0.019230 & 0.053260 & 1.160674 \\
\hline $\mathrm{C}$ & 0.217579 & -0.635556 & 2.402928 \\
\hline $\mathrm{C}$ & 1.478321 & -1.100825 & 2.658526 \\
\hline $\mathrm{C}$ & 2.572983 & -0.905746 & 1.735789 \\
\hline $\mathrm{C}$ & 2.399217 & -0.256549 & 0.541933 \\
\hline $\mathrm{C}$ & 1.090210 & 0.219223 & 0.206164 \\
\hline $\mathrm{H}$ & -0.604454 & -0.771548 & 3.108621 \\
\hline $\mathrm{H}$ & 1.679941 & -1.630305 & 3.592602 \\
\hline $\mathrm{H}$ & 3.559754 & -1.280134 & 2.015587 \\
\hline $\mathrm{H}$ & 3.237324 & -0.102644 & -0.140036 \\
\hline $\mathrm{C}$ & 0.582665 & 0.920154 & -0.870527 \\
\hline $\mathrm{N}$ & -1.154554 & 0.581208 & 0.759528 \\
\hline $\mathrm{C}$ & -0.883604 & 1.167281 & -0.554967 \\
\hline $\mathrm{C}$ & 1.313214 & 1.428786 & -2.026210 \\
\hline $\mathrm{C}$ & 2.273079 & 0.628839 & -2.686478 \\
\hline $\mathrm{C}$ & 2.960699 & 1.114734 & -3.798738 \\
\hline $\mathrm{C}$ & 2.714673 & 2.408721 & -4.271010 \\
\hline $\mathrm{C}$ & 1.769448 & 3.214191 & -3.624431 \\
\hline $\mathrm{C}$ & 1.070237 & 2.730365 & -2.518806 \\
\hline $\mathrm{H}$ & 2.444254 & -0.394815 & -2.350208 \\
\hline $\mathrm{H}$ & 3.685270 & 0.474587 & -4.306311 \\
\hline $\mathrm{H}$ & 3.256582 & 2.787568 & -5.140024 \\
\hline $\mathrm{H}$ & 1.574057 & 4.225920 & -3.985173 \\
\hline $\mathrm{H}$ & 0.337393 & 3.368745 & -2.019874 \\
\hline $\mathrm{C}$ & -1.916939 & 0.680204 & -1.585632 \\
\hline $\mathrm{C}$ & -1.626887 & 0.570990 & -2.952926 \\
\hline $\mathrm{C}$ & -2.604981 & 0.142289 & -3.857117 \\
\hline $\mathrm{C}$ & -3.886870 & -0.185951 & -3.410646 \\
\hline $\mathrm{C}$ & -4.183793 & -0.079134 & -2.048202 \\
\hline $\mathrm{C}$ & -3.209504 & 0.351143 & -1.143802 \\
\hline $\mathrm{H}$ & -0.635230 & 0.816414 & -3.328096 \\
\hline $\mathrm{H}$ & -2.355329 & 0.061352 & -4.916685 \\
\hline $\mathrm{H}$ & -4.649000 & -0.522060 & -4.116922 \\
\hline $\mathrm{H}$ & -5.178844 & -0.338459 & -1.681357 \\
\hline
\end{tabular}




\begin{tabular}{|c|c|c|c|}
\hline $\mathrm{H}$ & -3.436134 & 0.422103 & -0.080432 \\
\hline $\mathrm{H}$ & -1.023915 & 2.264271 & -0.442521 \\
\hline & & & \\
\hline \multicolumn{4}{|l|}{46} \\
\hline $\mathrm{N}$ & 0.173729 & -0.845140 & 0.169734 \\
\hline $\mathrm{C}$ & 0.173184 & -0.567801 & 1.544993 \\
\hline $\mathrm{C}$ & 1.442857 & -0.134532 & 1.923313 \\
\hline $\mathrm{C}$ & 2.265248 & -0.156923 & 0.739597 \\
\hline $\mathrm{C}$ & 1.448683 & -0.608783 & -0.336803 \\
\hline $\mathrm{C}$ & 1.948456 & -0.778052 & -1.633588 \\
\hline $\mathrm{C}$ & 3.604739 & 0.191297 & 0.478545 \\
\hline $\mathrm{C}$ & 1.859307 & 0.348115 & 3.250617 \\
\hline $\mathrm{C}$ & -1.037969 & -0.713495 & 2.365422 \\
\hline $\mathrm{C}$ & -0.966805 & -1.353875 & 3.617242 \\
\hline $\mathrm{C}$ & -2.104040 & -1.479285 & 4.416309 \\
\hline $\mathrm{C}$ & -3.332631 & -0.971100 & 3.978663 \\
\hline $\mathrm{C}$ & -3.414967 & -0.335429 & 2.734733 \\
\hline $\mathrm{C}$ & -2.278891 & -0.206417 & 1.933956 \\
\hline $\mathrm{H}$ & -0.011436 & -1.757342 & 3.956961 \\
\hline $\mathrm{H}$ & -2.032554 & -1.981687 & 5.383199 \\
\hline $\mathrm{H}$ & -4.222506 & -1.071589 & 4.603364 \\
\hline $\mathrm{H}$ & -4.368483 & 0.067929 & 2.387576 \\
\hline $\mathrm{H}$ & -2.350049 & 0.300341 & 0.970105 \\
\hline $\mathrm{C}$ & 3.070393 & -0.089467 & 3.822554 \\
\hline $\mathrm{C}$ & 3.479868 & 0.371849 & 5.076625 \\
\hline $\mathrm{C}$ & 2.687677 & 1.280138 & 5.787216 \\
\hline $\mathrm{C}$ & 1.483362 & 1.725882 & 5.229204 \\
\hline $\mathrm{C}$ & 1.076401 & 1.269634 & 3.974125 \\
\hline $\mathrm{H}$ & 3.684722 & -0.814576 & 3.285229 \\
\hline $\mathrm{H}$ & 4.419782 & 0.014486 & 5.503033 \\
\hline $\mathrm{H}$ & 3.007022 & 1.639621 & 6.767427 \\
\hline $\mathrm{H}$ & 0.861059 & 2.441391 & 5.771329 \\
\hline $\mathrm{H}$ & 0.142875 & 1.632000 & 3.540384 \\
\hline $\mathrm{C}$ & -0.868818 & -1.491292 & -0.562233 \\
\hline $\mathrm{C}$ & 4.101797 & 0.041880 & -0.813969 \\
\hline $\mathrm{C}$ & 3.284568 & -0.446214 & -1.856831 \\
\hline $\mathrm{H}$ & 1.316507 & -1.148050 & -2.441674 \\
\hline $\mathrm{H}$ & 3.703232 & -0.564191 & -2.858080 \\
\hline $\mathrm{H}$ & 5.139928 & 0.304946 & -1.026842 \\
\hline $\mathrm{H}$ & 4.244736 & 0.565720 & 1.279369 \\
\hline $\mathrm{C}$ & -1.378311 & -0.886352 & -1.718461 \\
\hline $\mathrm{C}$ & -2.387851 & -1.523626 & -2.445097 \\
\hline $\mathrm{C}$ & -2.897469 & -2.753140 & -2.013774 \\
\hline $\mathrm{C}$ & -2.389189 & -3.349210 & -0.854043 \\
\hline $\mathrm{C}$ & -1.369625 & -2.726530 & -0.130846 \\
\hline $\mathrm{H}$ & -0.993275 & 0.084811 & -2.033298 \\
\hline $\mathrm{H}$ & -2.784448 & -1.050609 & -3.345293 \\
\hline $\mathrm{H}$ & -3.690074 & -3.245886 & -2.579657 \\
\hline $\mathrm{H}$ & -2.780960 & -4.309576 & -0.513797 \\
\hline $\mathrm{H}$ & -0.961416 & -3.188454 & 0.769092 \\
\hline
\end{tabular}




\section{3. ${ }^{1} \mathrm{H}$ and ${ }^{13} \mathrm{C}$ NMR Spectra of $\mathrm{N}$-Unsubstituted indoles}

\section{2,3-Diphenyl-1H-indole (3a)}
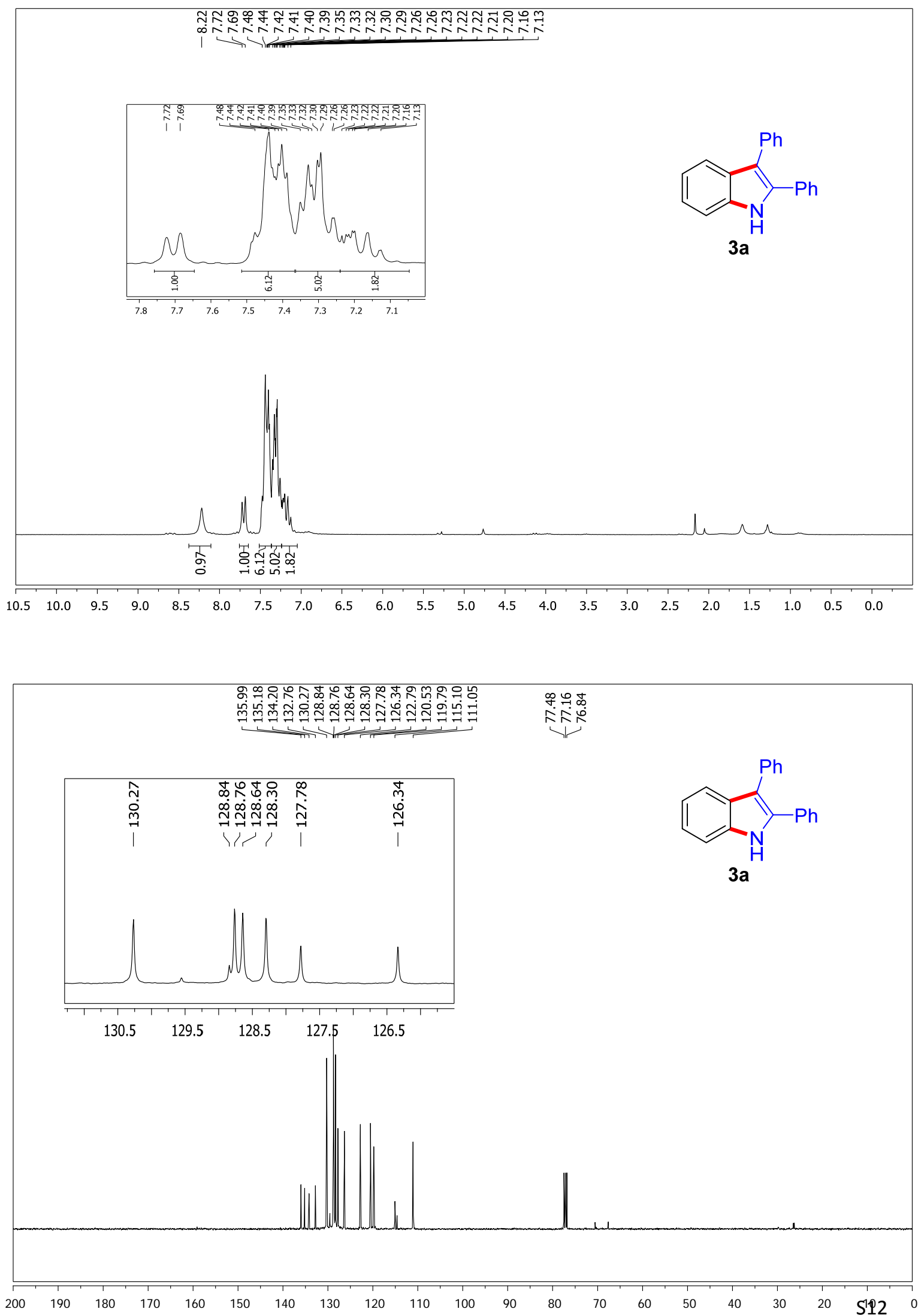


\section{2,3-Di-p-tolyl-1H-indole (3b)}
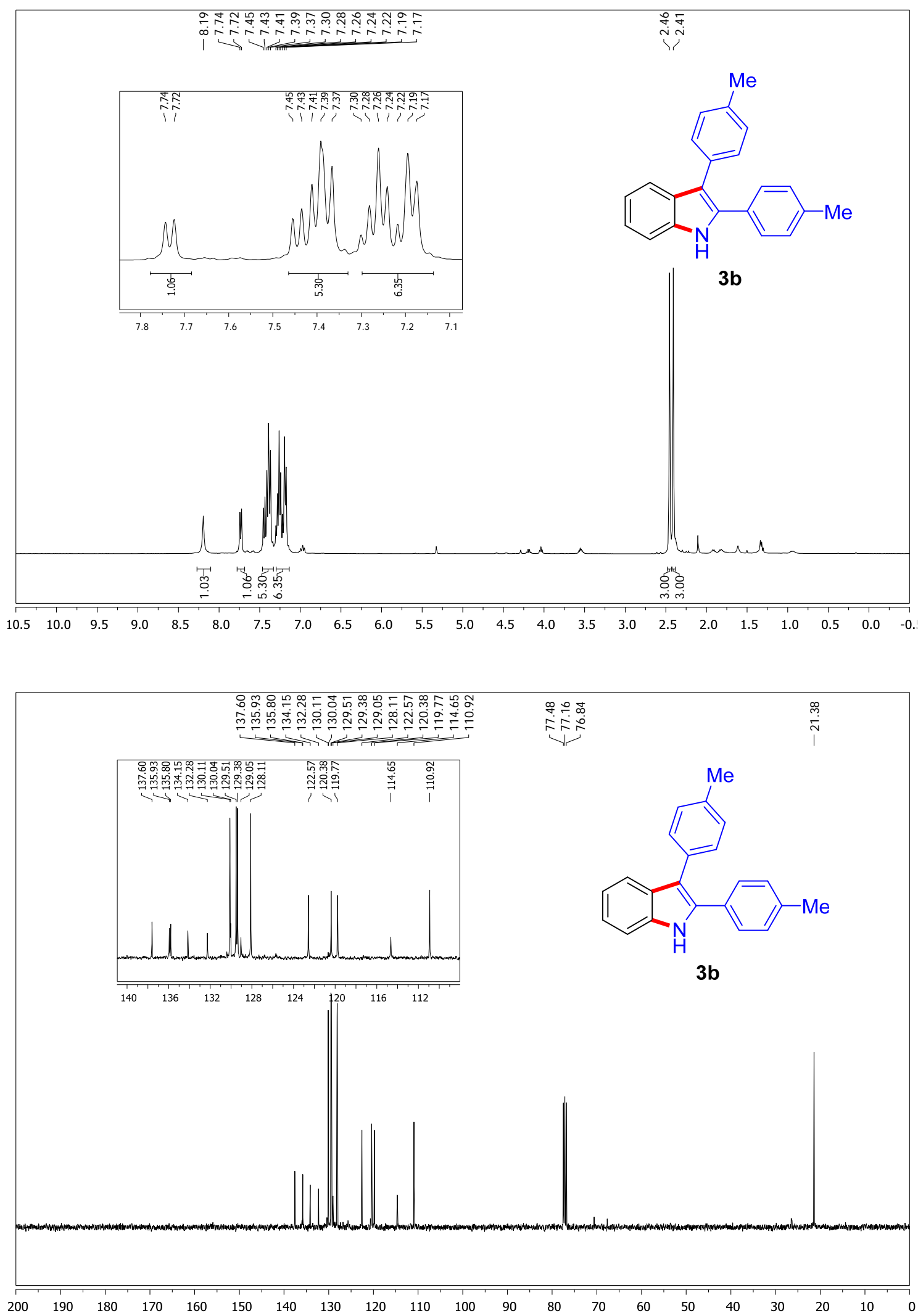
2,3-Bis(4-bromophenyl)-1H-indole (3c)

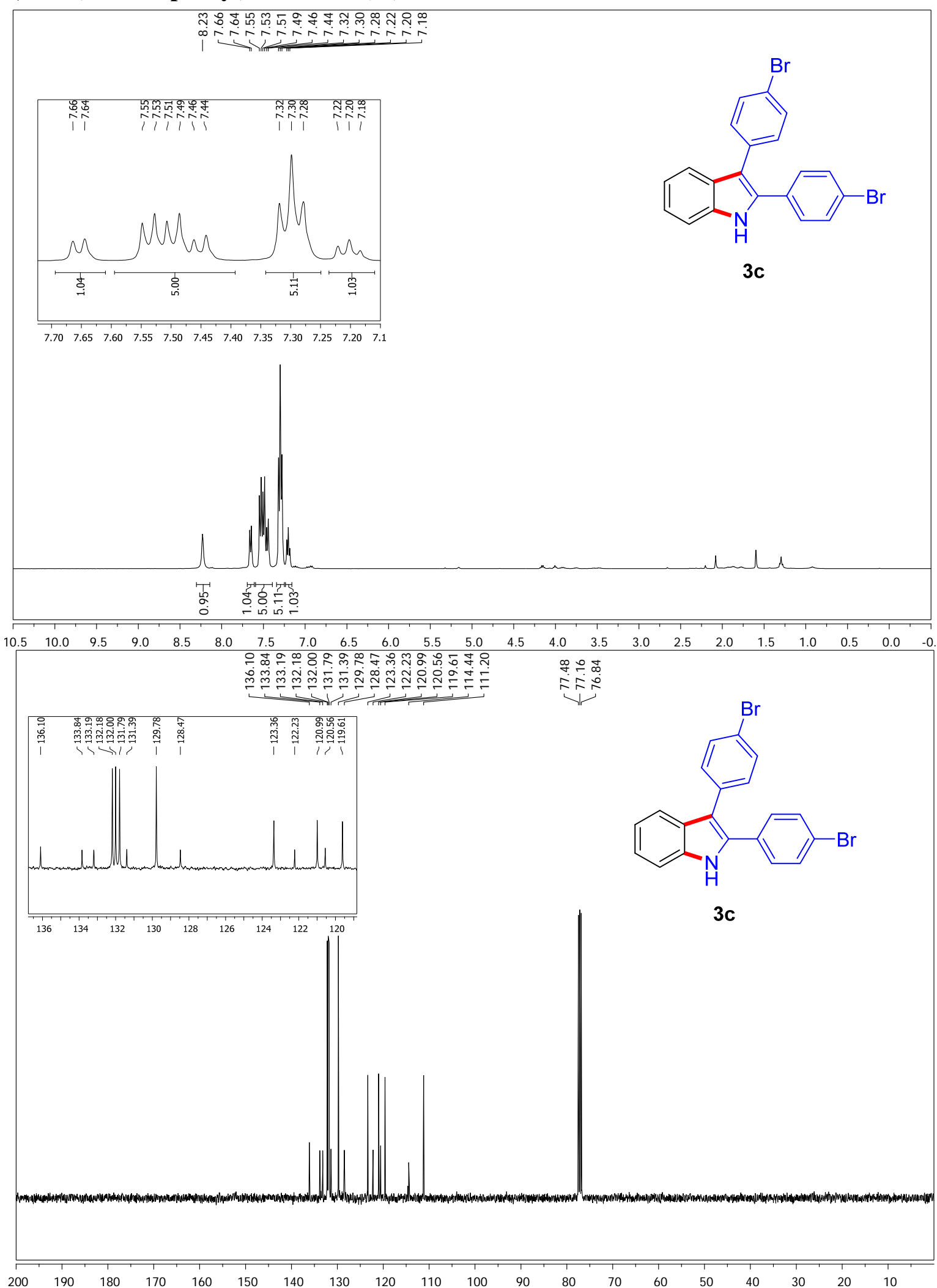


2,3-Bis(4-chlorophenyl)-1H-indole (3d)
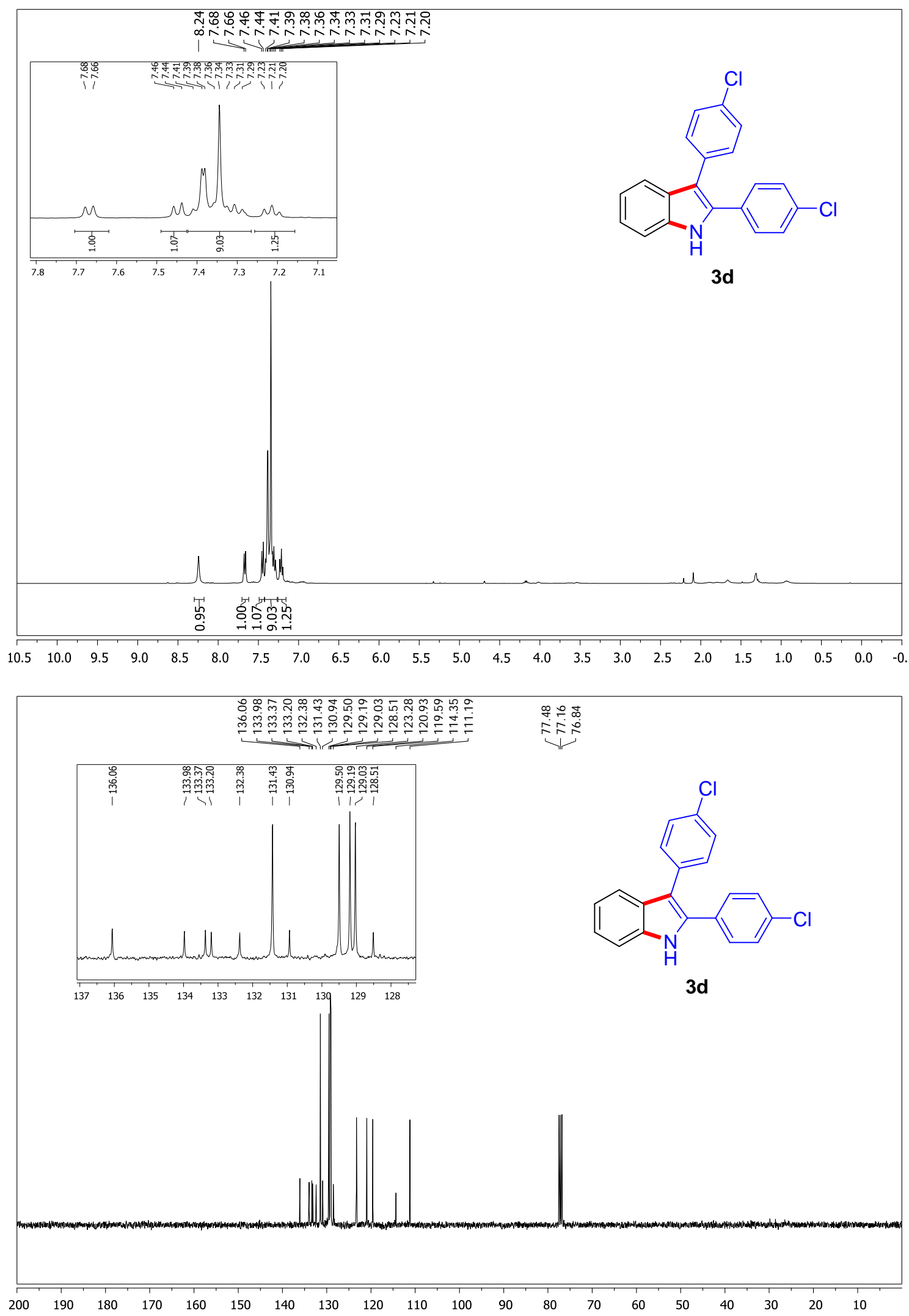


\section{2,3-Bis(4-fluorophenyl)-1H-indole (3e)}
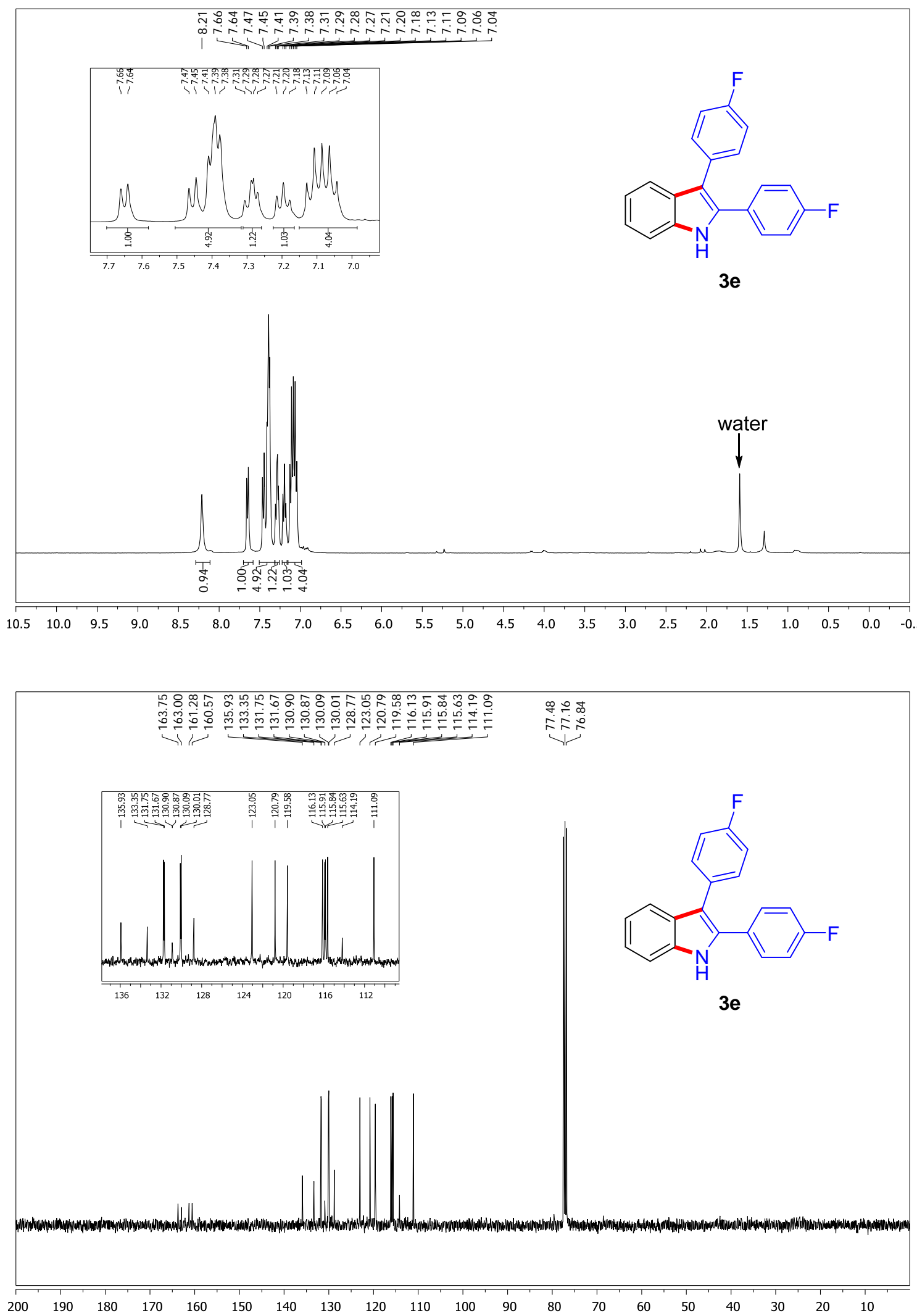


\section{2,3-Bis(4-(trifluoromethyl)phenyl)-1H-indole (3f)}
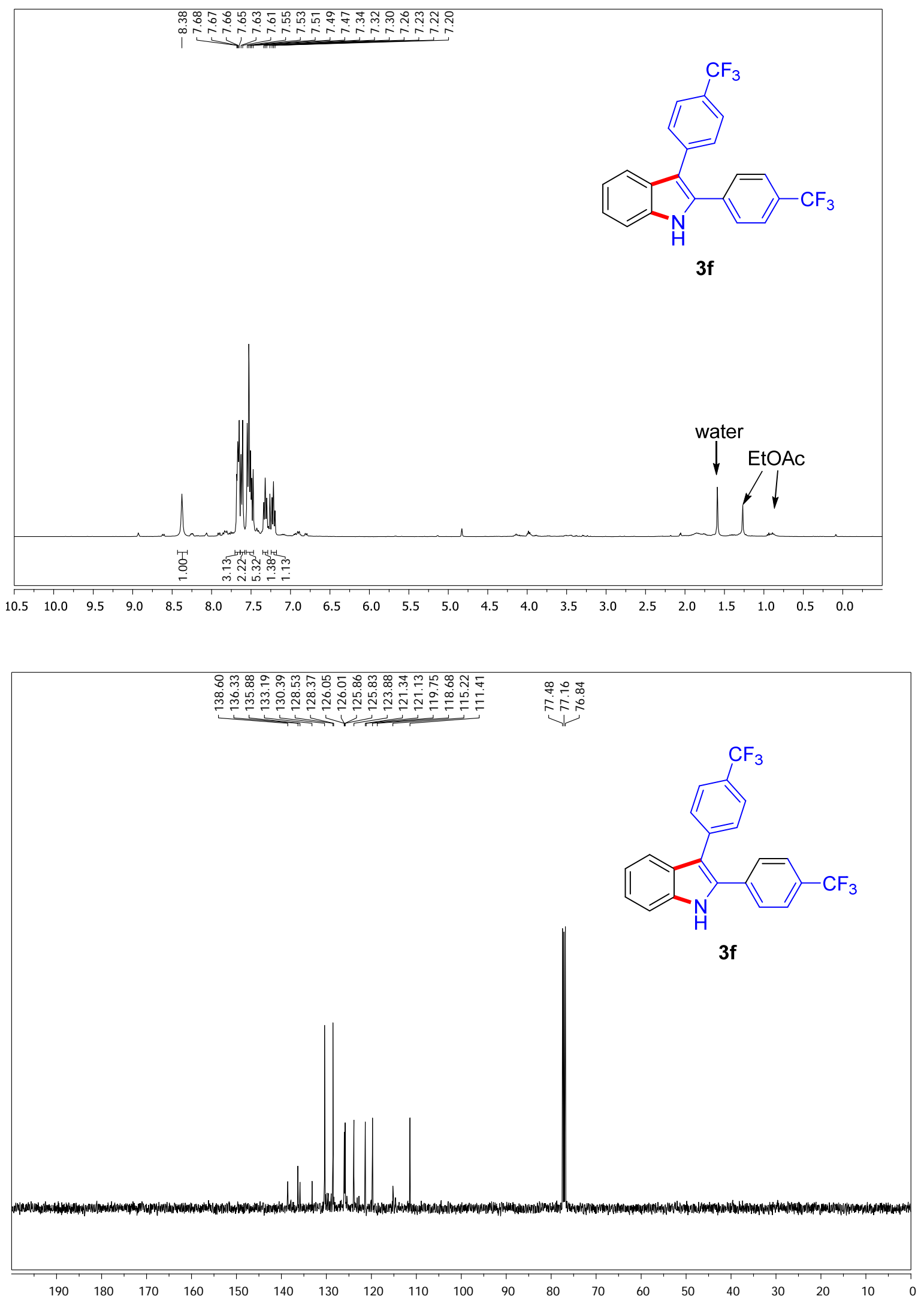


\section{2,3-Bis(3-methoxyphenyl)-1H-indole (3g)}
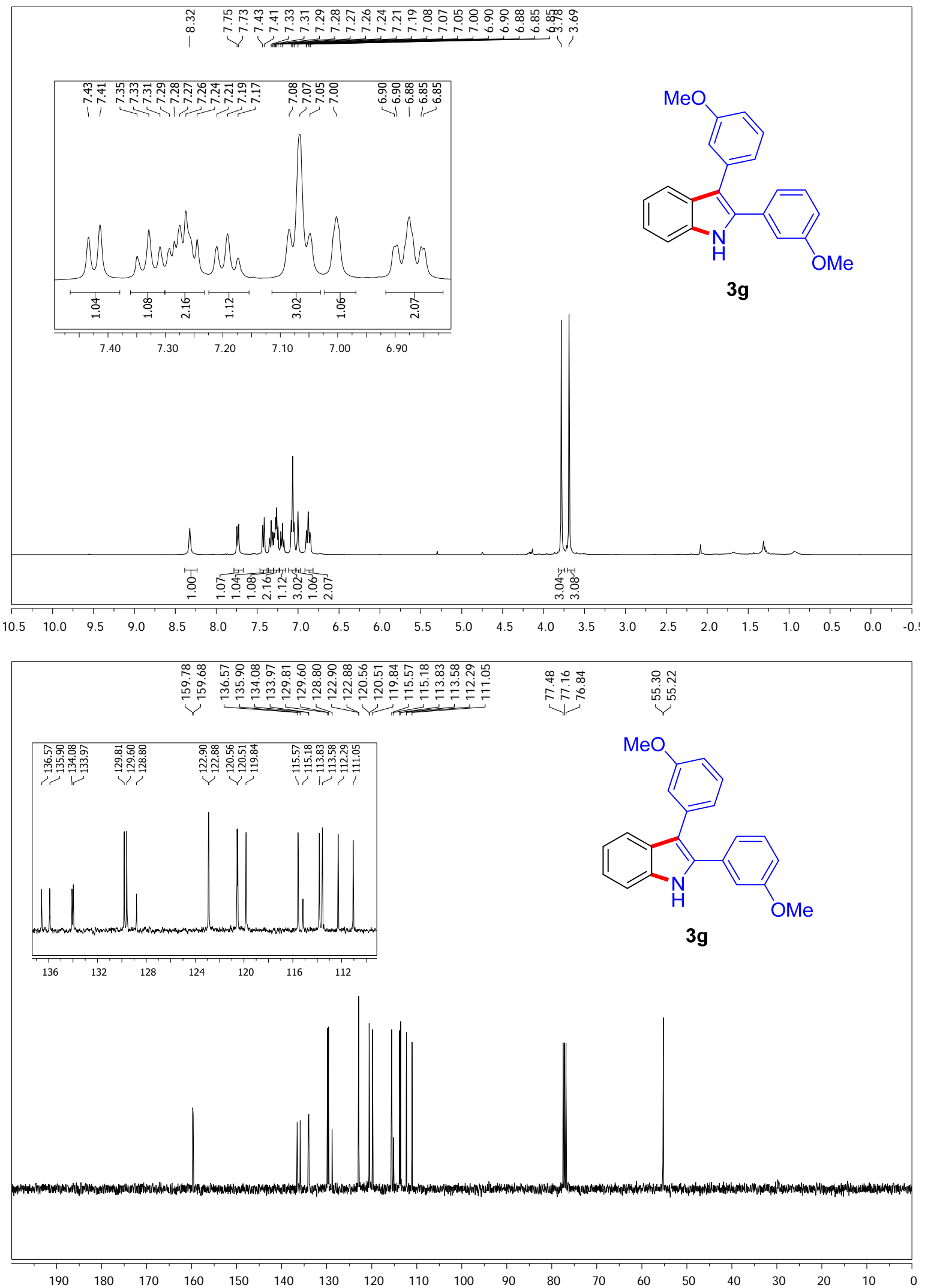
2,3-Bis(3-chlorophenyl)-1H-indole (3h)
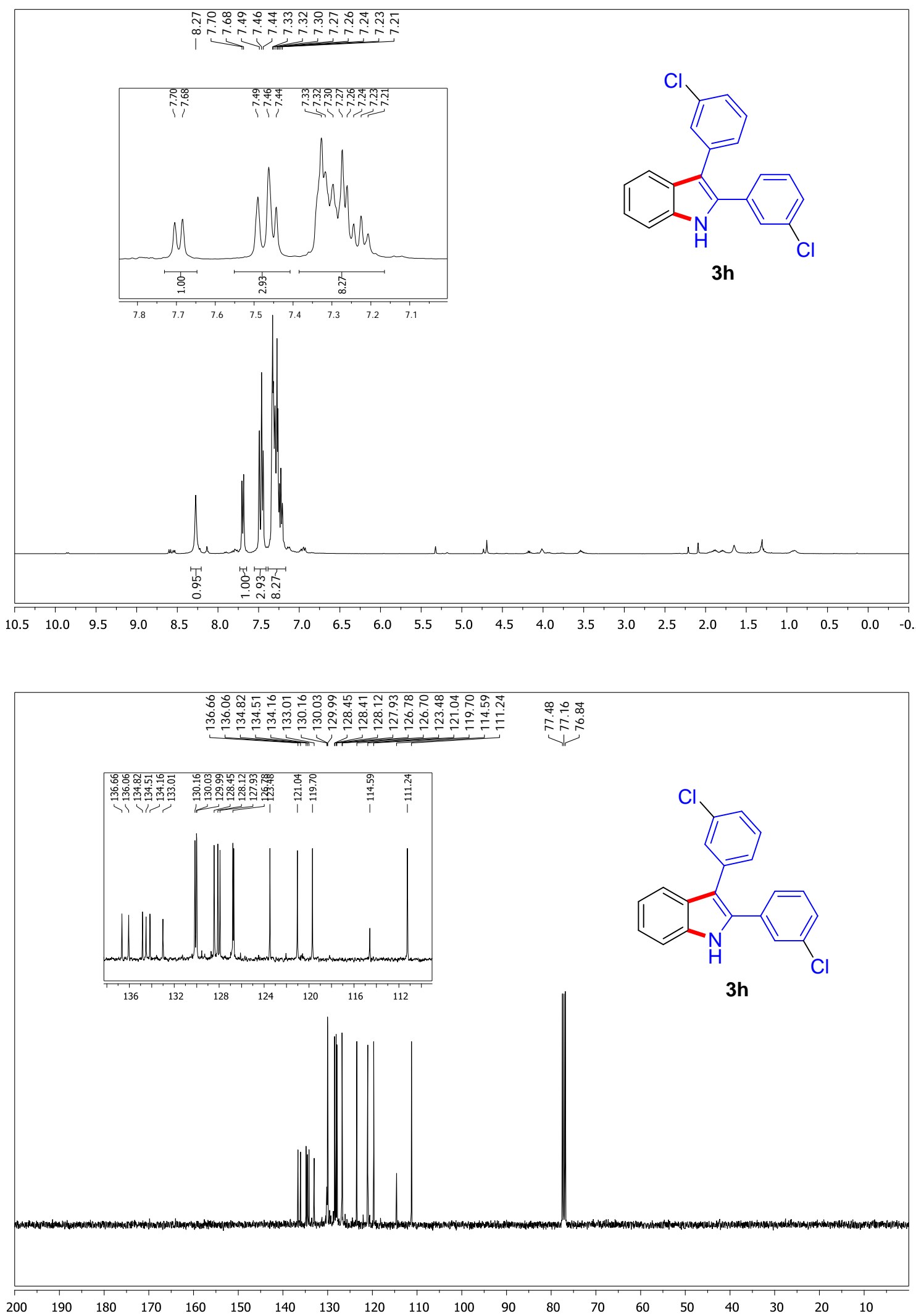


\section{2,3-Bis(2-chlorophenyl)-1H-indole (3i)}
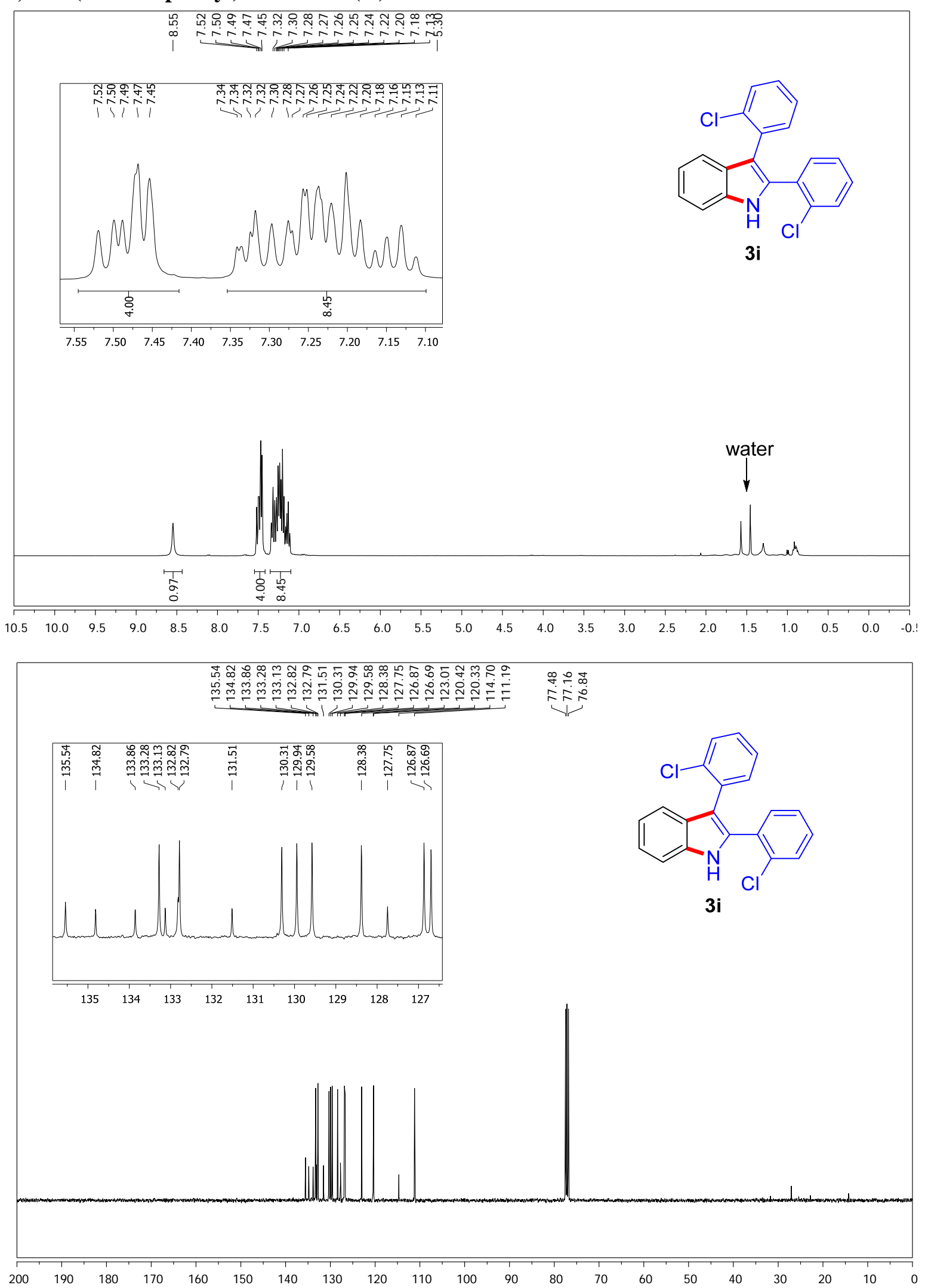


\section{2,3-Bis(3,4-dichlorophenyl)-1H-indole (3j)}
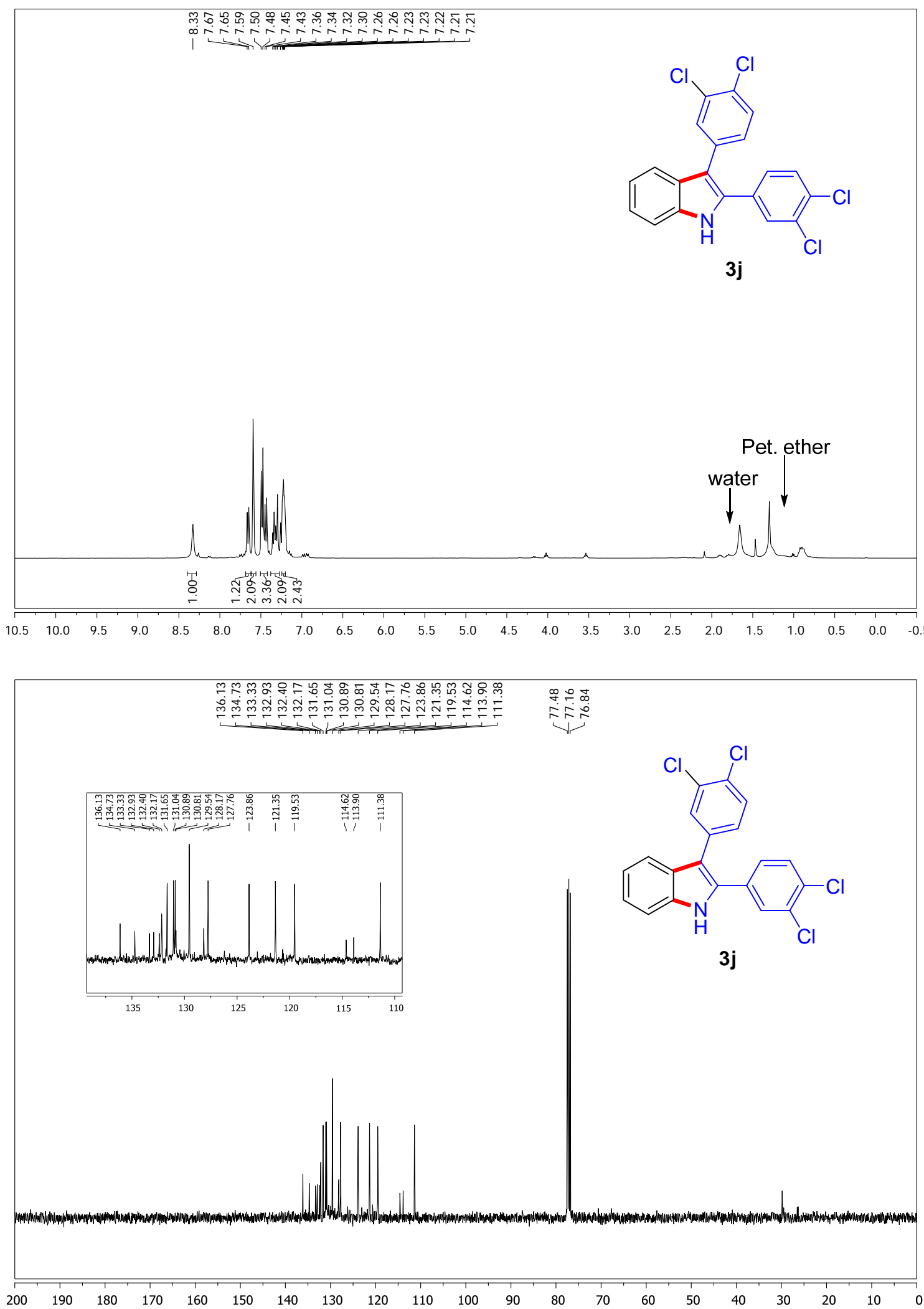
3-Phenyl-2-(p-tolyl)-1H-indole (3k) and 2-Phenyl-3-(p-tolyl)-1H-indole (3k')

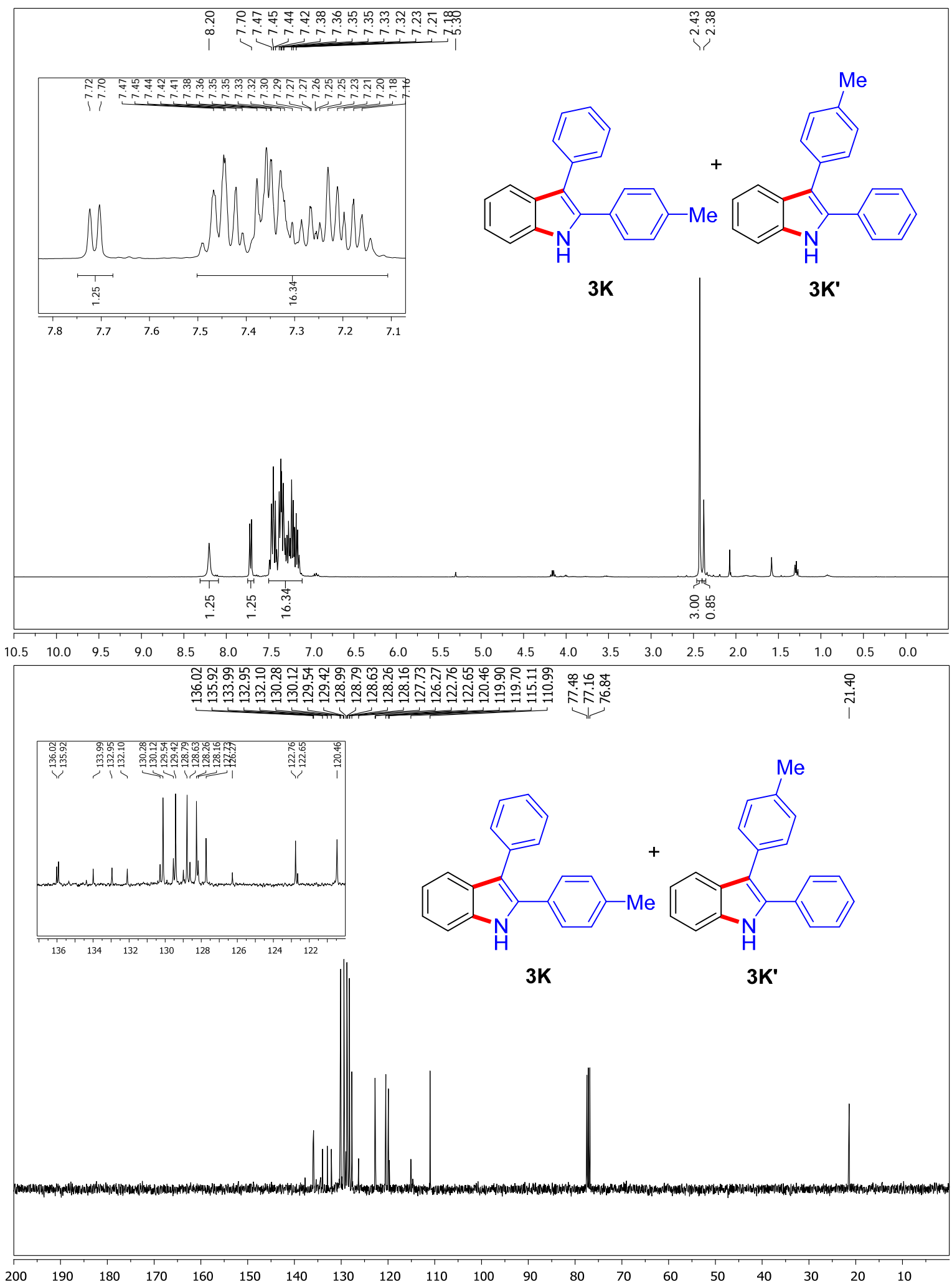


2-(4-Nitrophenyl)-3-phenyl-1H-indole (3l) and 3-(4-Nitrophenyl)-2-phenyl-1H-indole (3l')
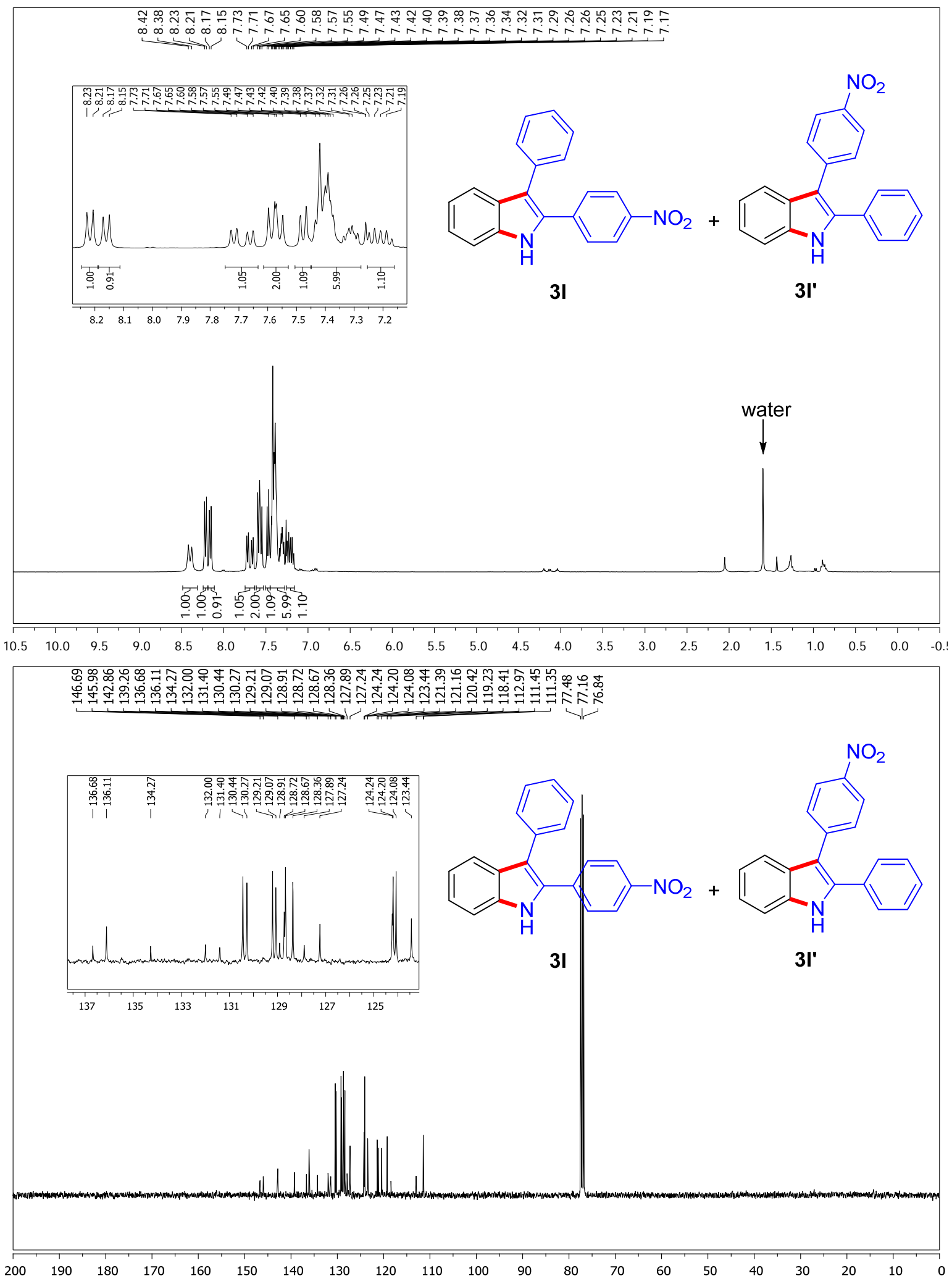
3-(4-Bromophenyl)-2-(4-nitrophenyl)-1H-indole (3m) and 2-(4-Bromophenyl)-3-(4nitrophenyl)-1H-indole ( $\left.3 \mathrm{~m}^{\prime}\right)$
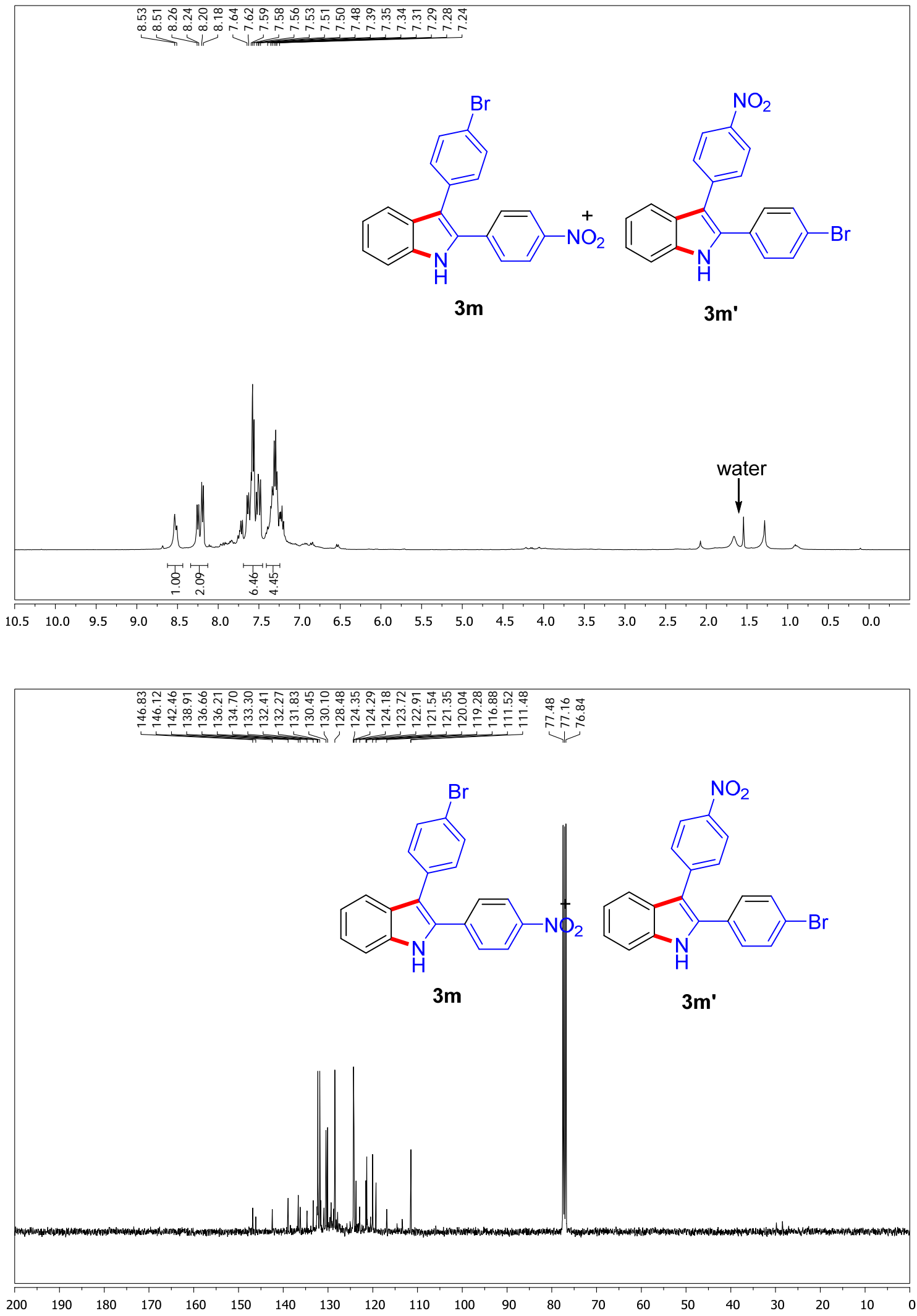
2-Methyl-3-phenyl-1H-indole (3n)
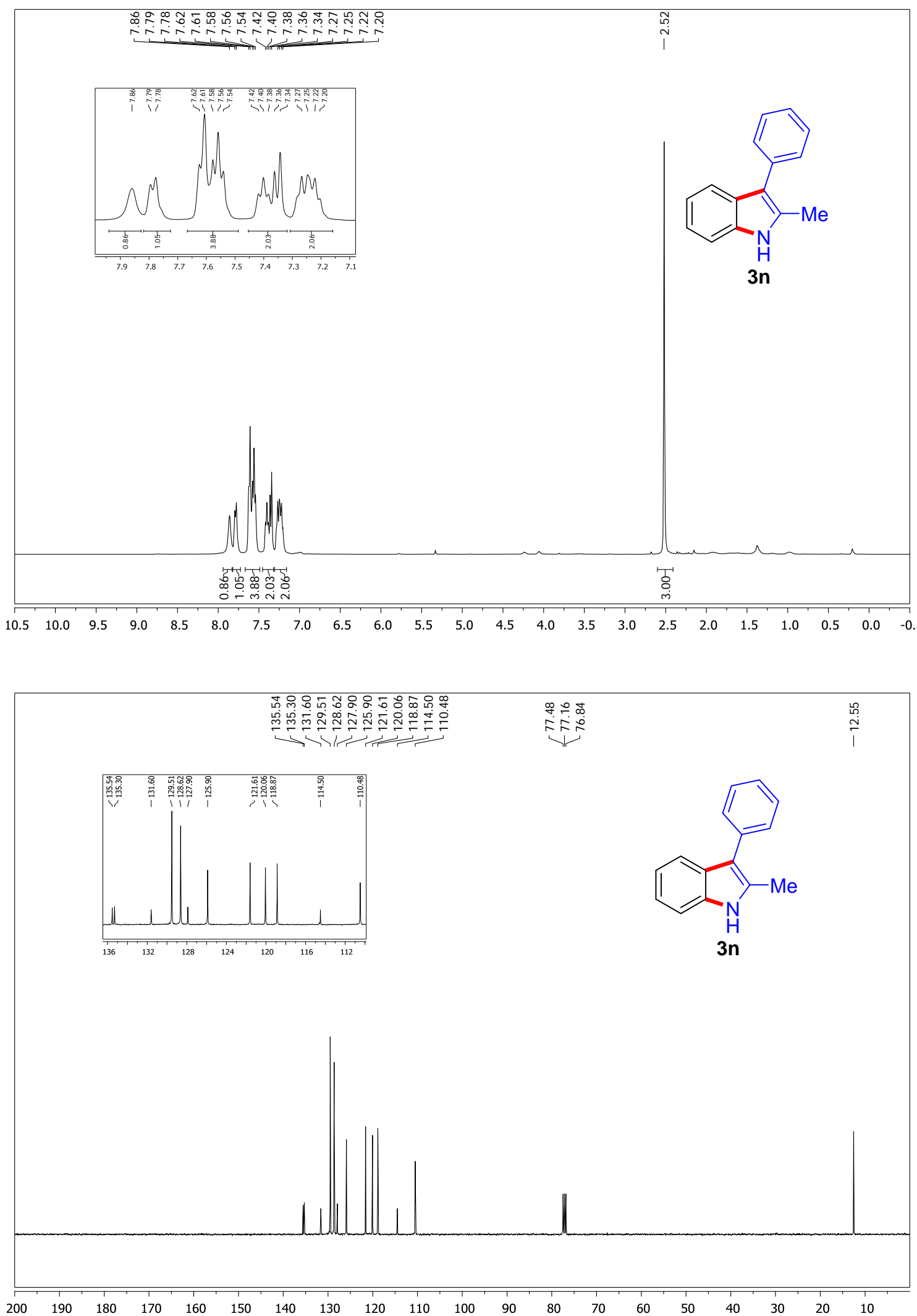


\section{4,7-Dimethyl-2,3-diphenyl-1H-indole (3o)}
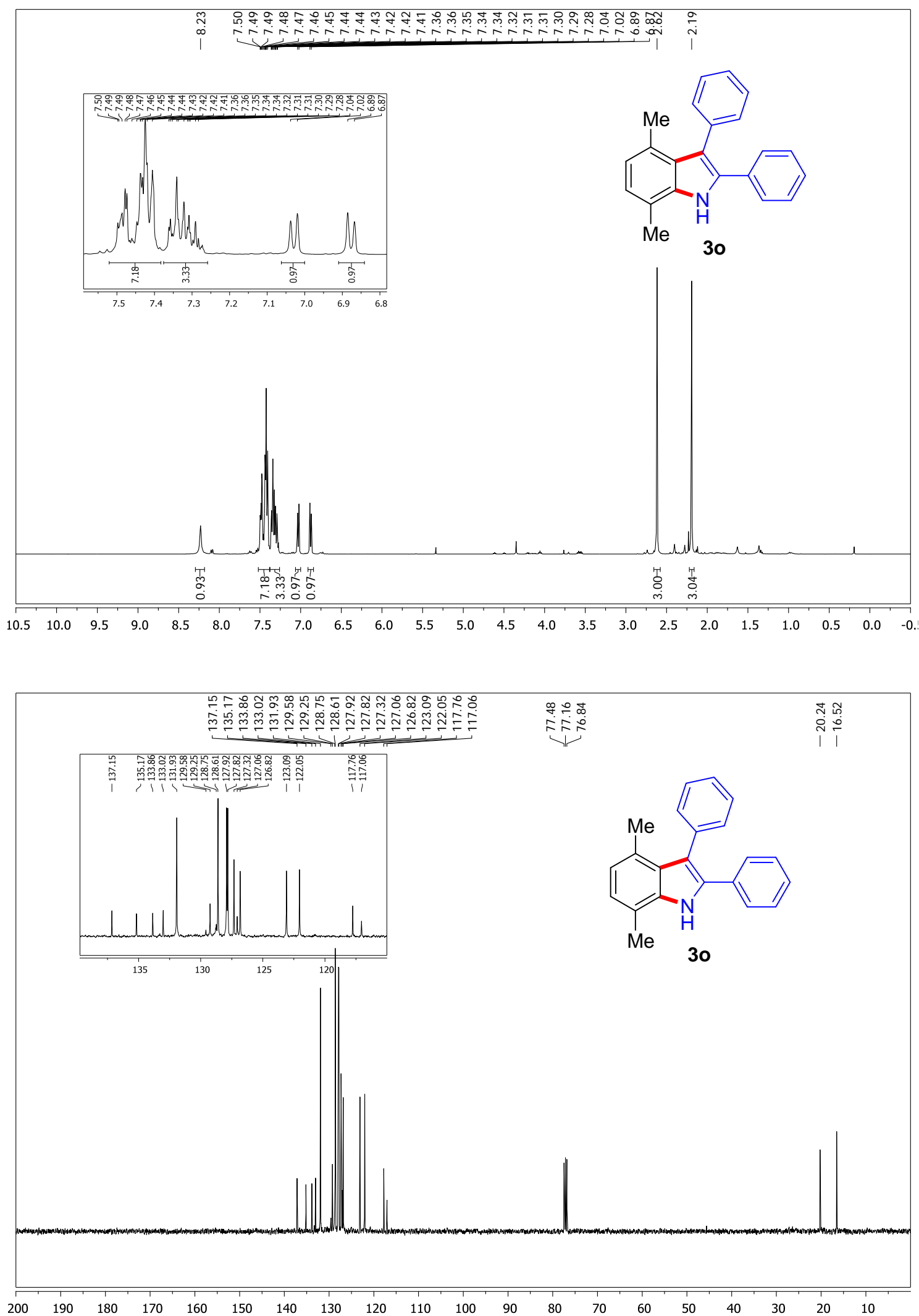


\section{2,3-Diphenyl-1H-benzo[f]indole (3p)}
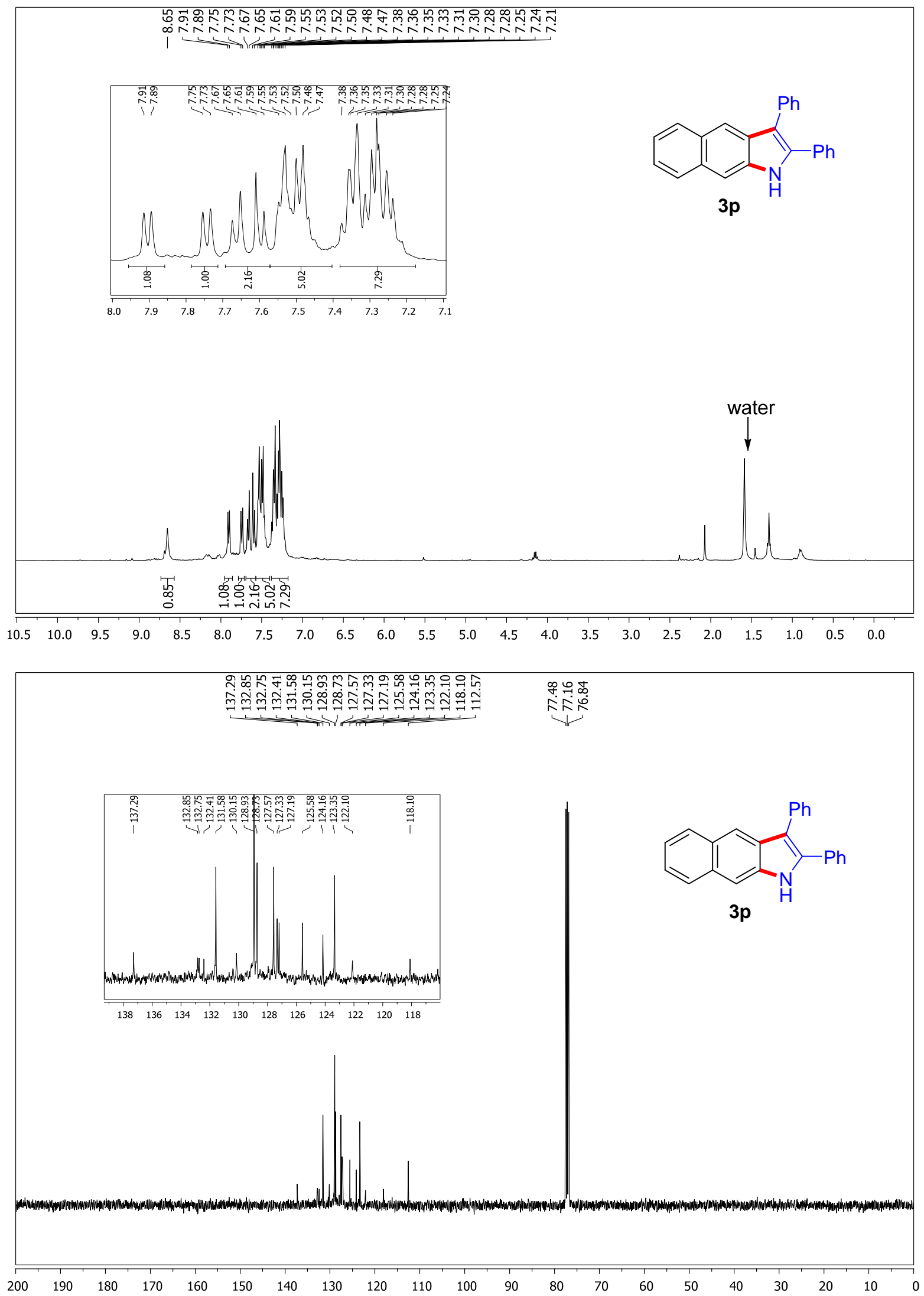
6,7-Diphenyl-5H-[1,3]dioxolo[4,5-f]indole (3q)
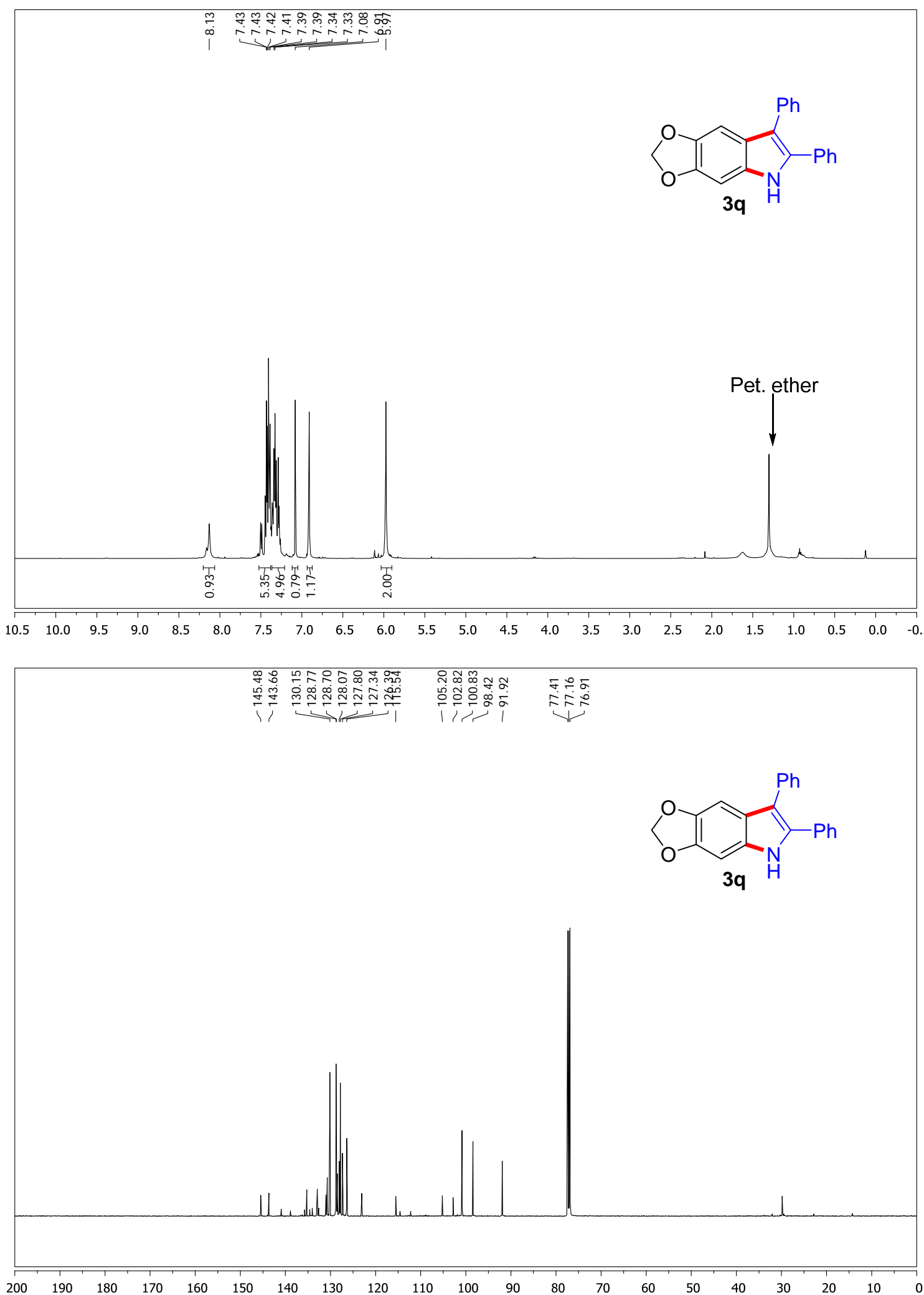


\section{4. ${ }^{1} \mathrm{H}$ and ${ }^{13} \mathrm{C}$ NMR Spectra of $\mathrm{N}$-Aryl Indoles}

\section{1,2,3-Triphenyl-1H-indole (4a)}
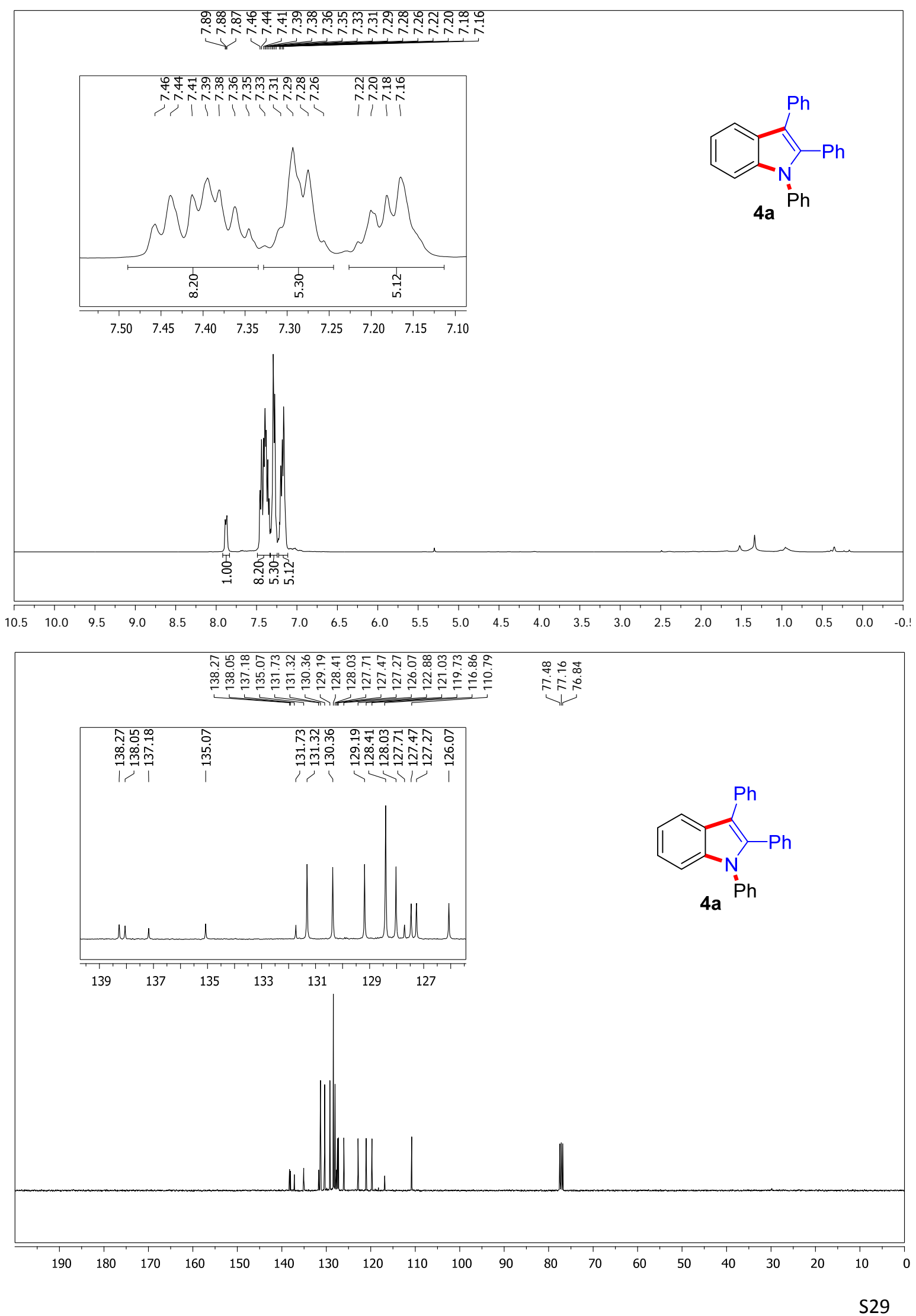
1-Phenyl-2,3-di-p-tolyl-1H-indole (4b)
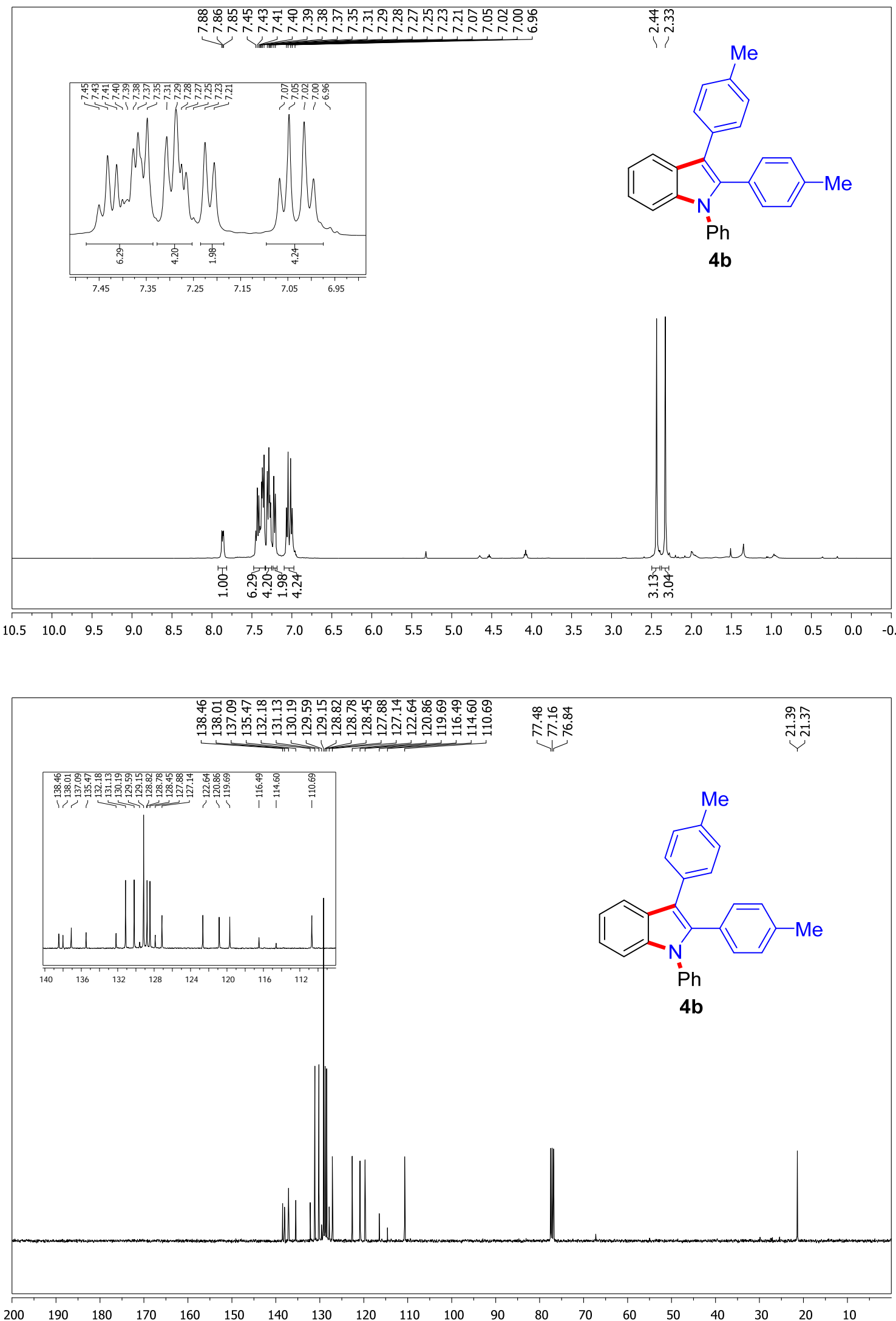
2,3-Bis(4-bromophenyl)-1-phenyl-1H-indole (4c)
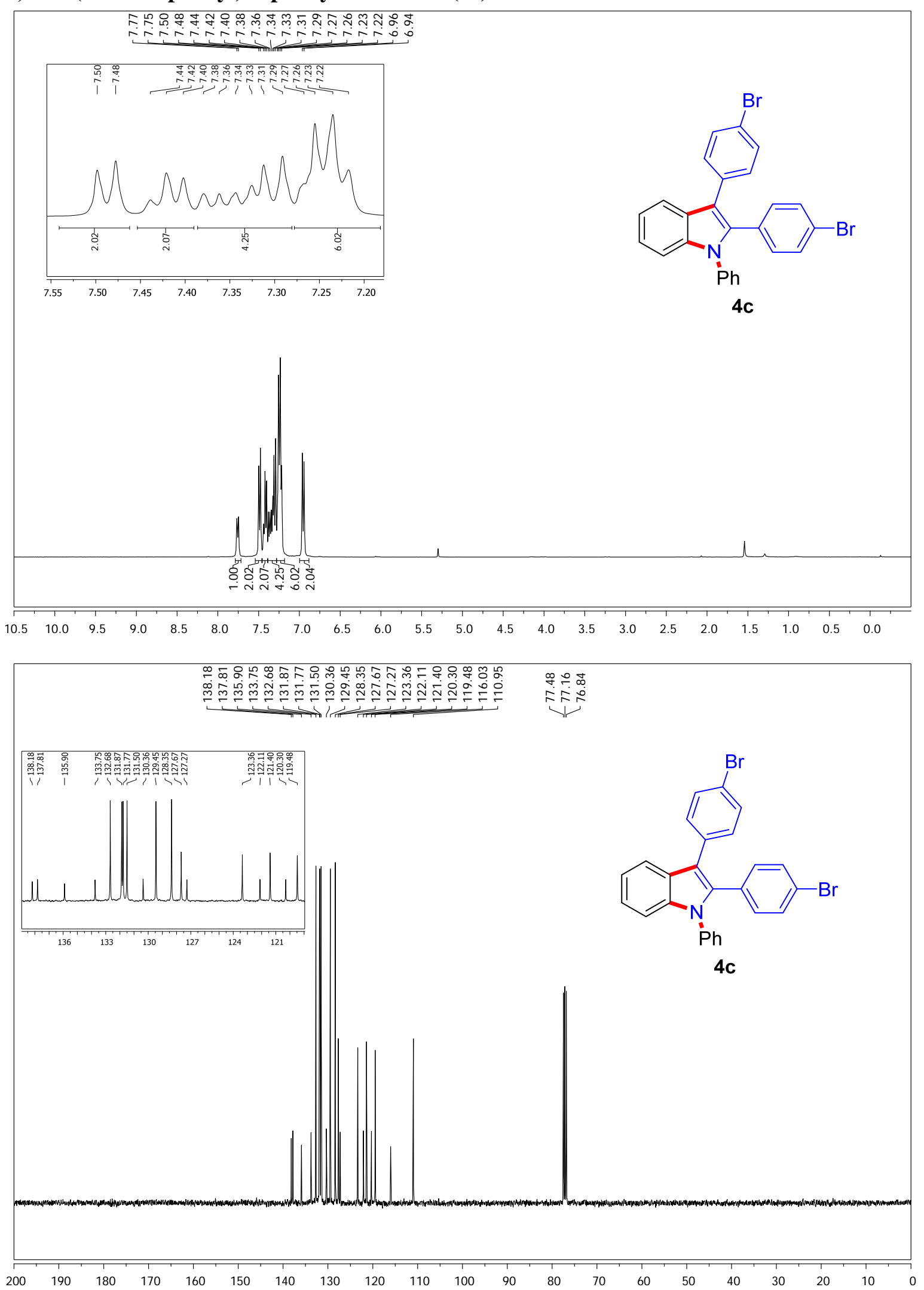


\section{2,3-Bis(4-chlorophenyl)-1-phenyl-1H-indole (4d)}
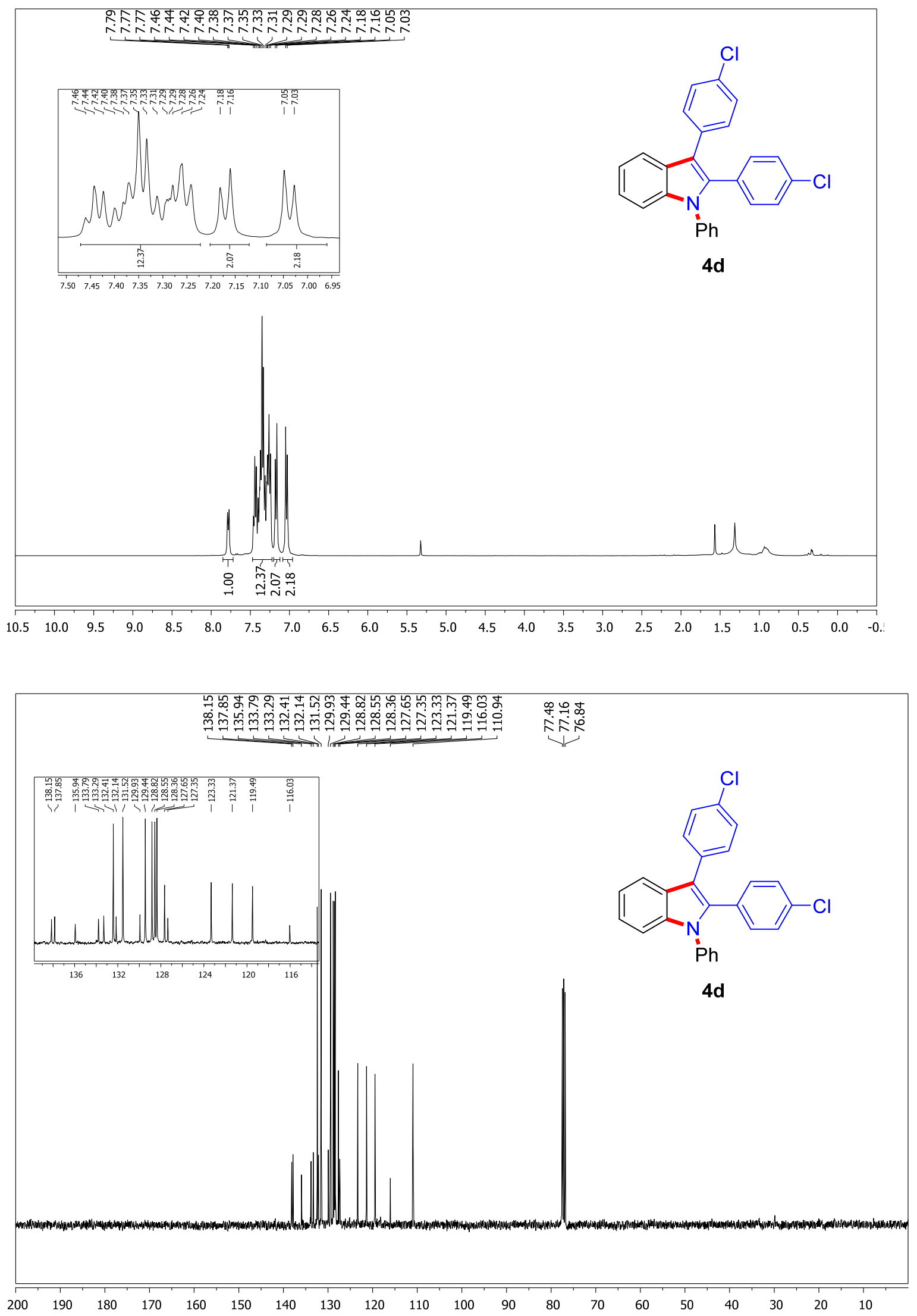


\section{2,3-Bis(4-fluorophenyl)-1-phenyl-1H-indole (4e)}
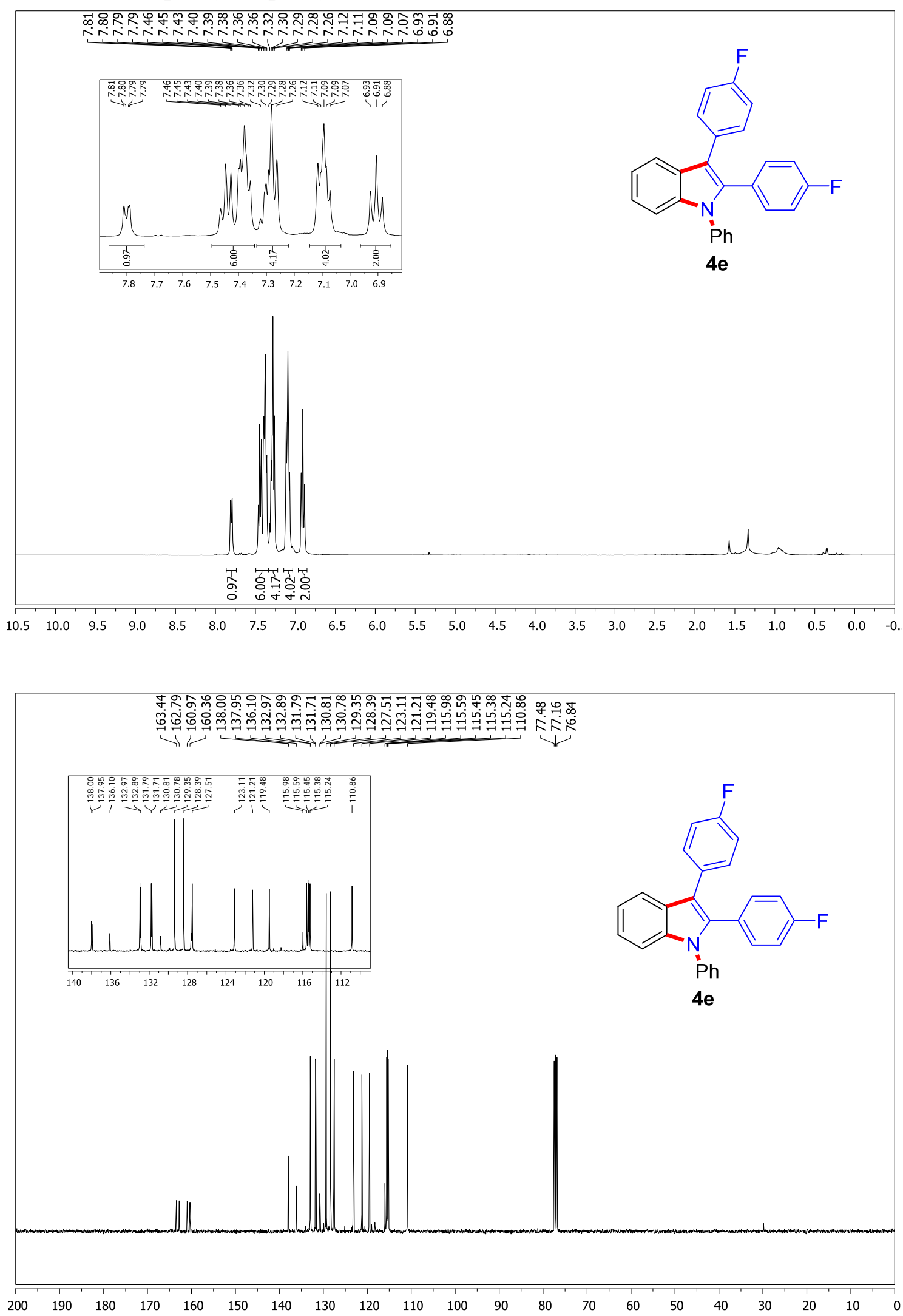
1-Phenyl-2,3-bis(4-(trifluoromethyl)phenyl)-1H-indole (4f)
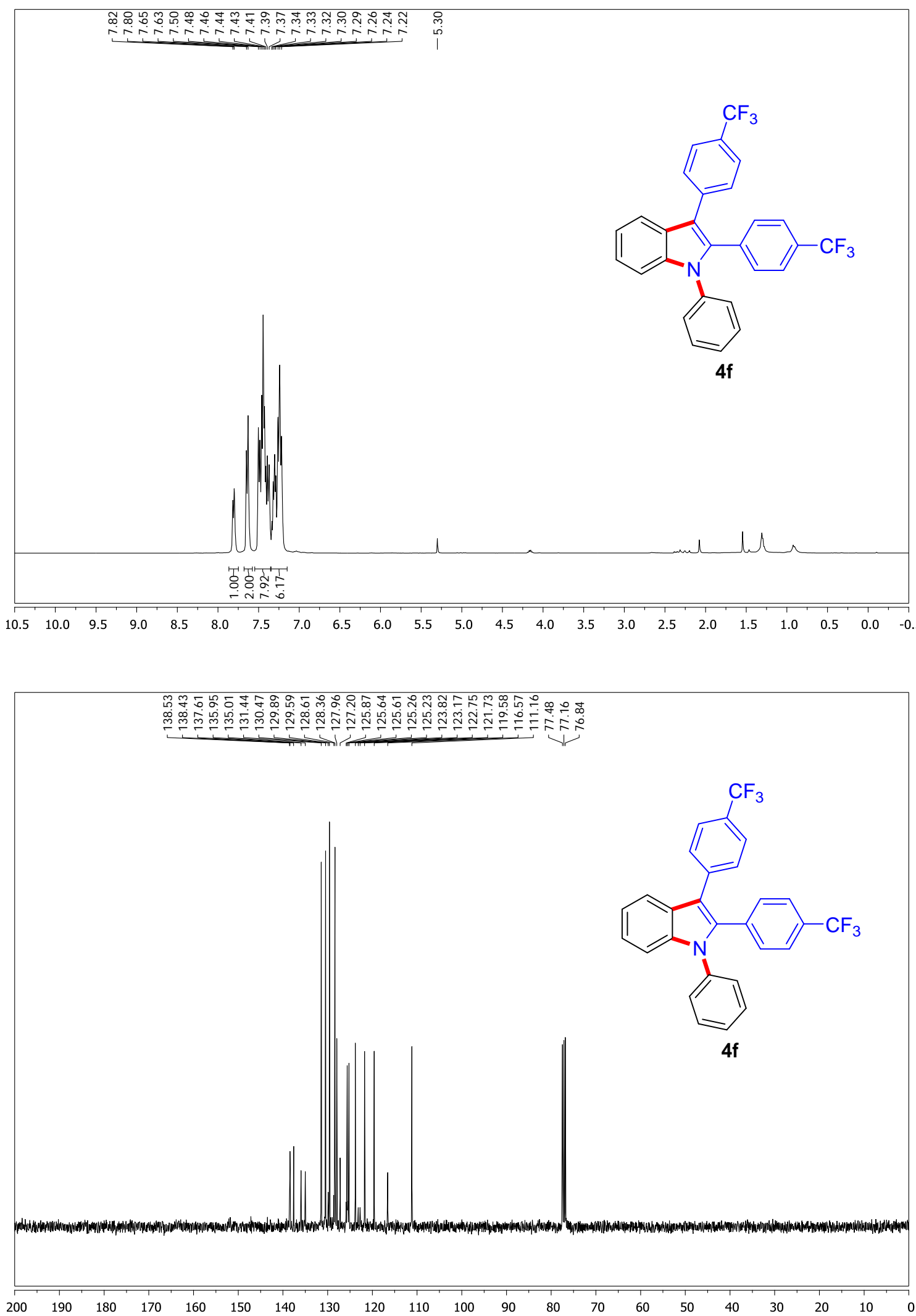


\section{2,3-Bis(3-methoxyphenyl)-1-phenyl-1H-indole (4g)}

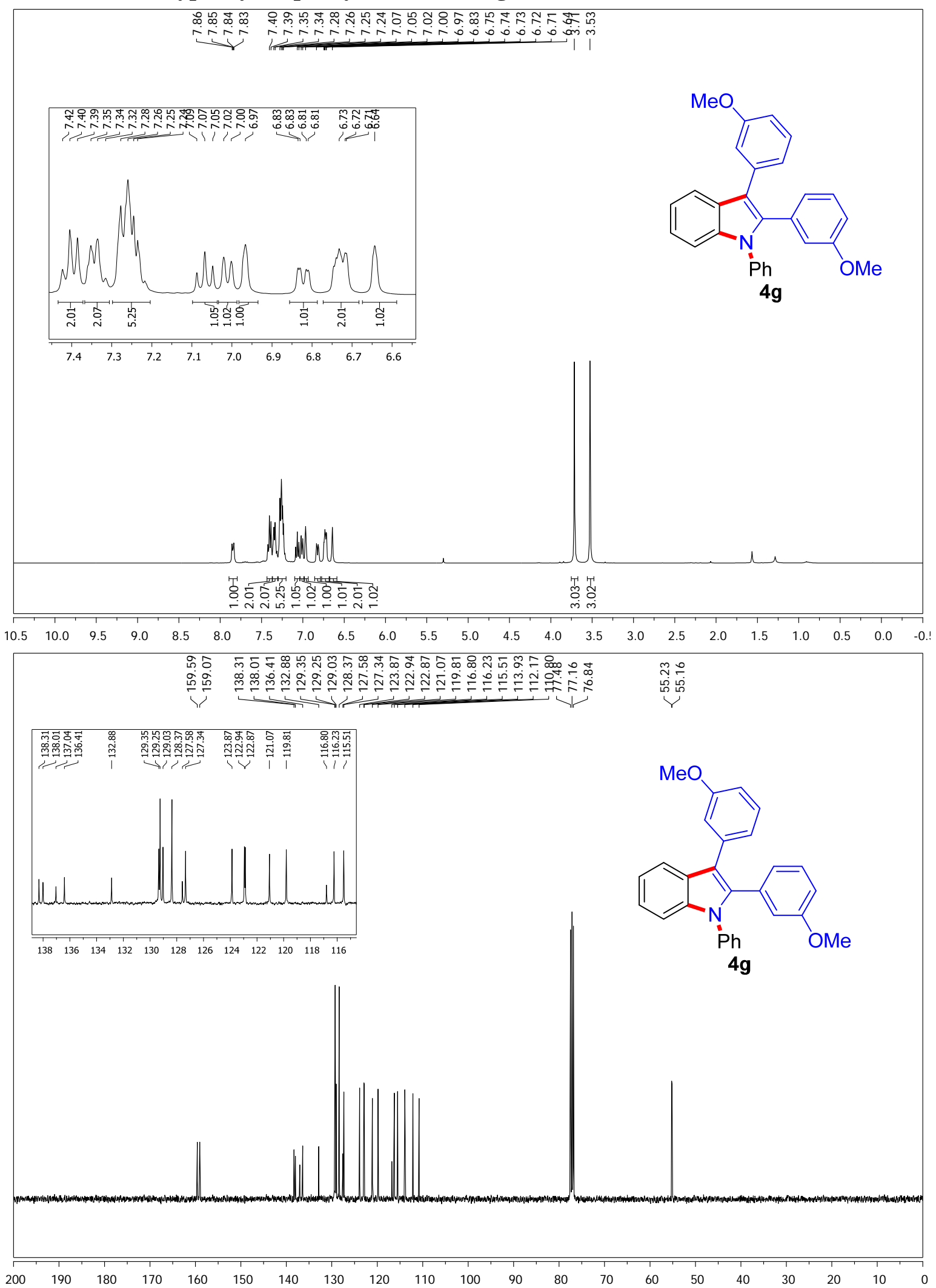




\section{2,3-Bis(3-chlorophenyl)-1-phenyl-1H-indole (4h)}
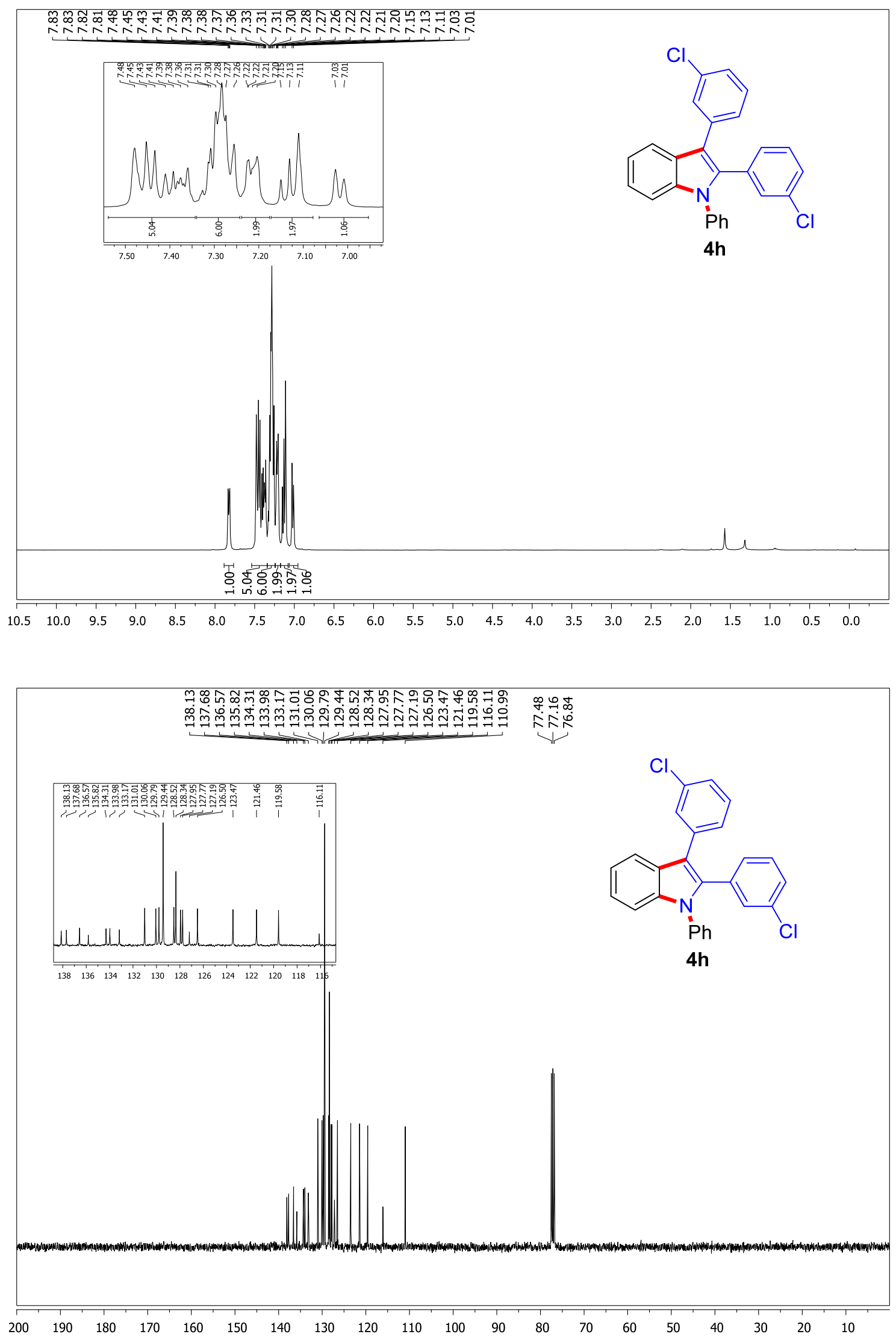


\section{2,3-Bis(2-chlorophenyl)-1-phenyl-1H-indole (4i)}
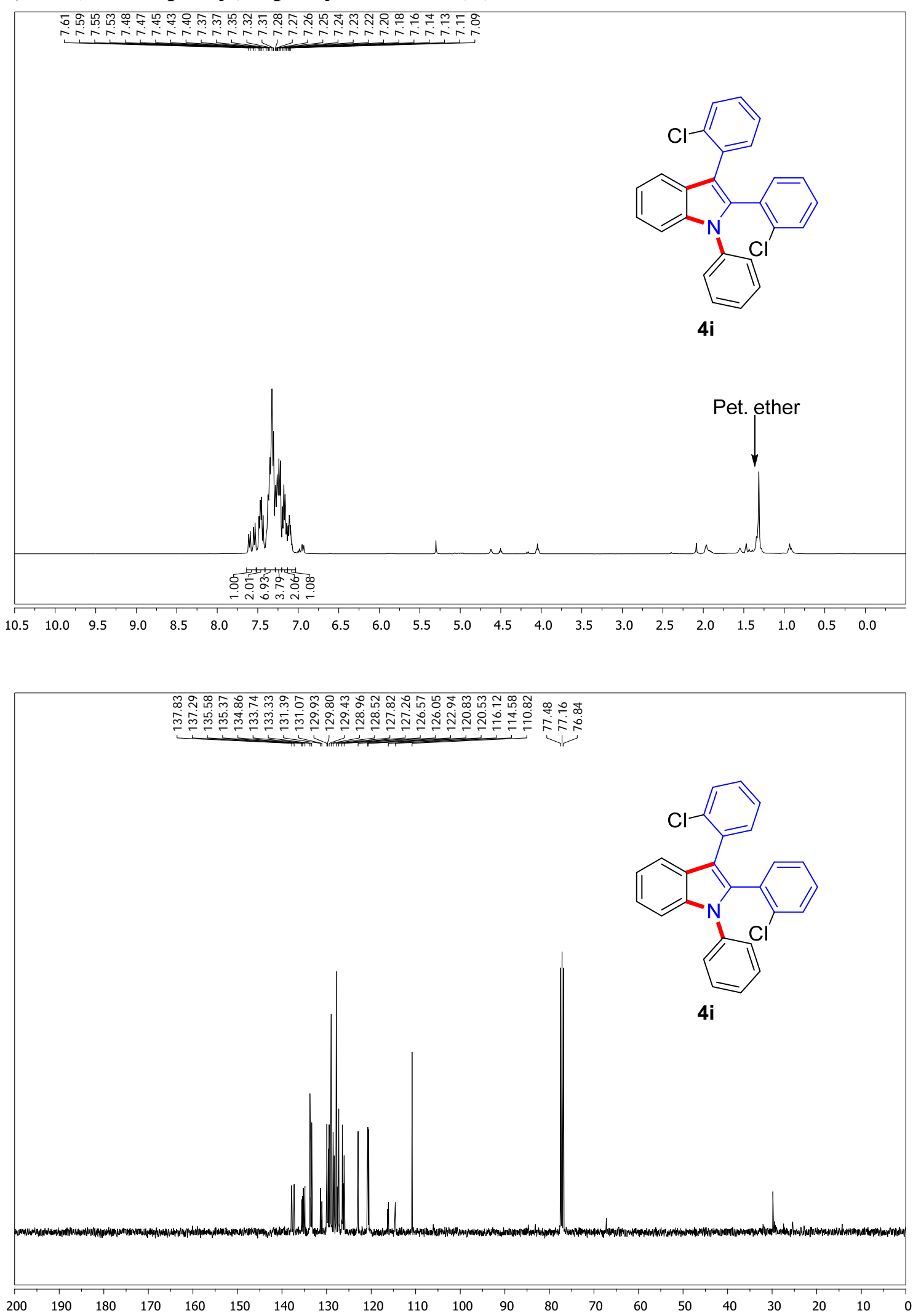


\section{2,3-Bis(3,4-dichlorophenyl)-1-phenyl-1H-indole (4j)}
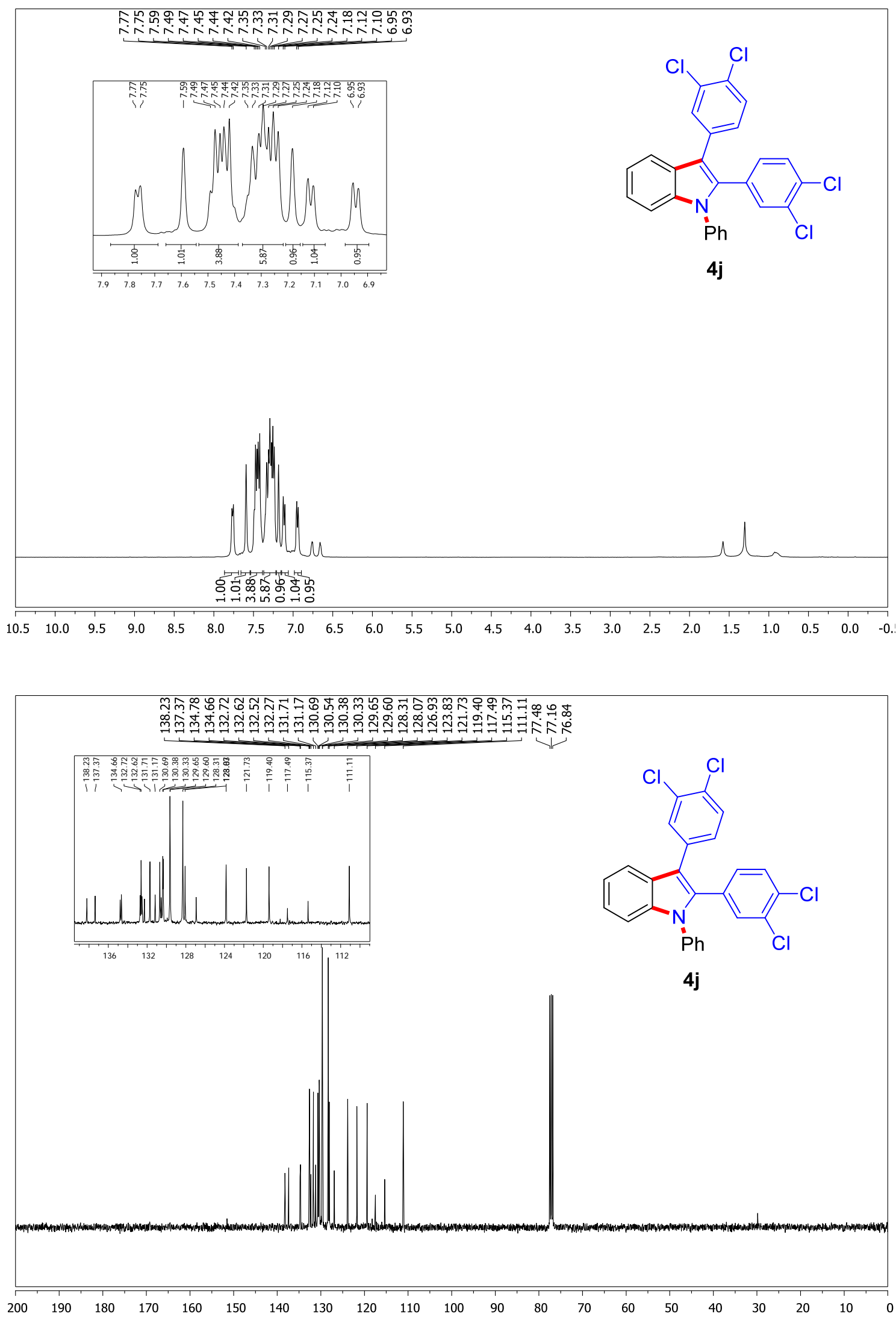
1,2-Diphenyl-3-(p-tolyl)-1H-indole (4k) and 1,3-Diphenyl-2-(p-tolyl)-1H-indole (4k')

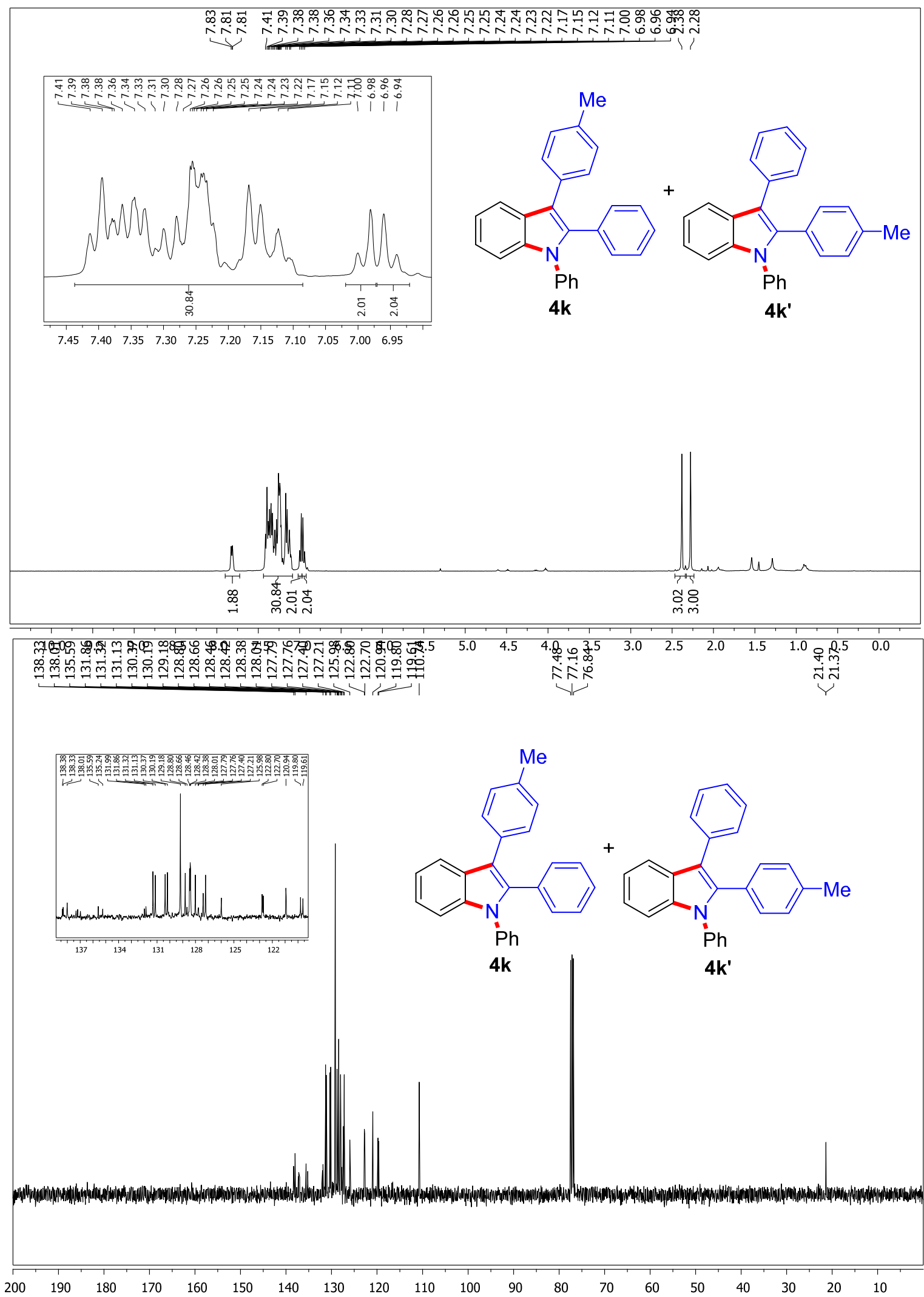


2-(4-Nitrophenyl)-1,3-diphenyl-1H-indole (4l) and 3-(4-Nitrophenyl)-1,2-diphenyl-1Hindole (4l')
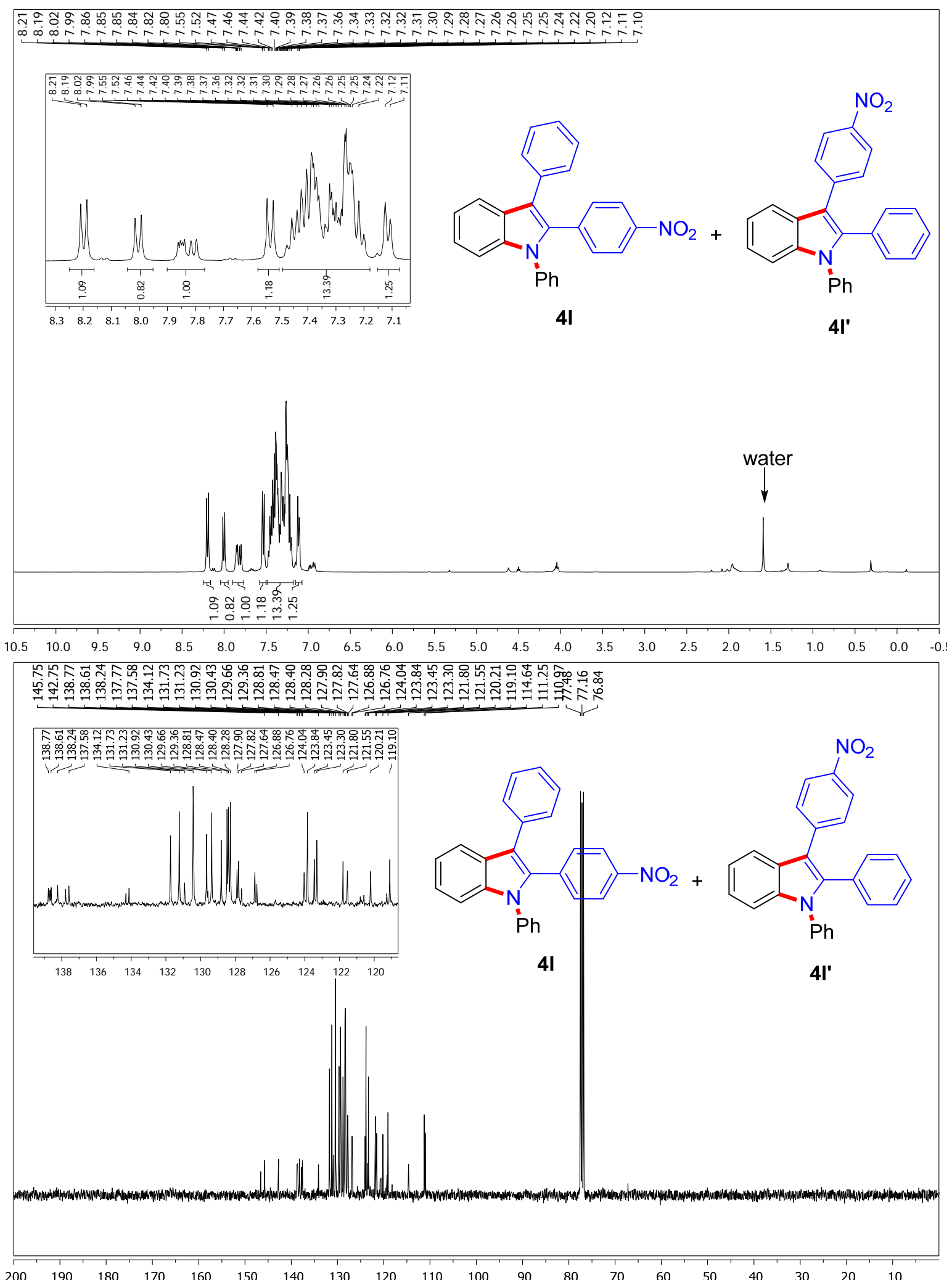
3-(4-Bromophenyl)-2-(4-nitrophenyl)-1-phenyl-1H-indole (4m) and 2-(4-Bromophenyl)3-(4-nitrophenyl)-1-phenyl-1H-indole (4m')
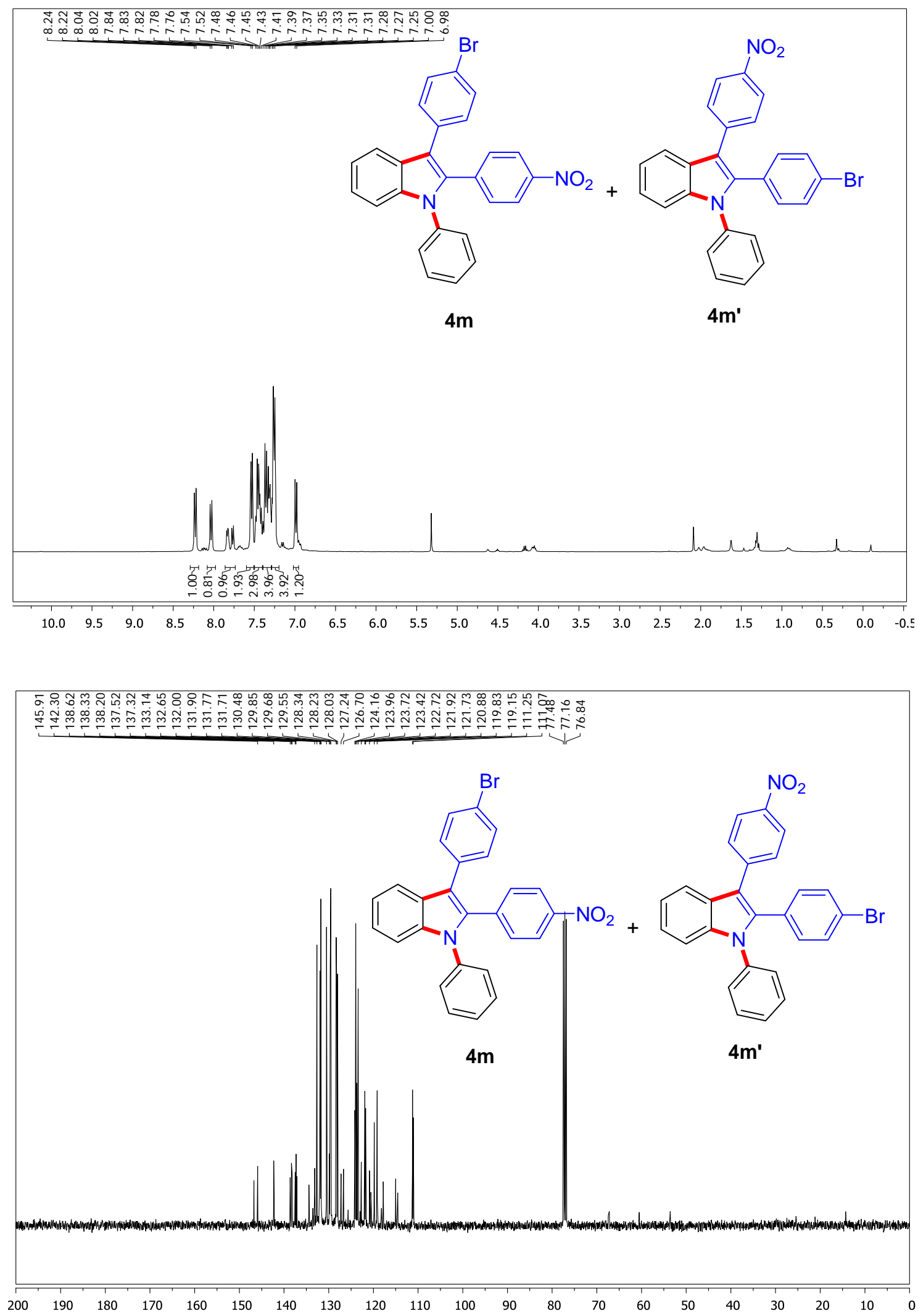
2-Methyl-1,3-diphenyl-1H-indole (4n) and 3-Methyl-1,2-diphenyl-1H-indole (4n')
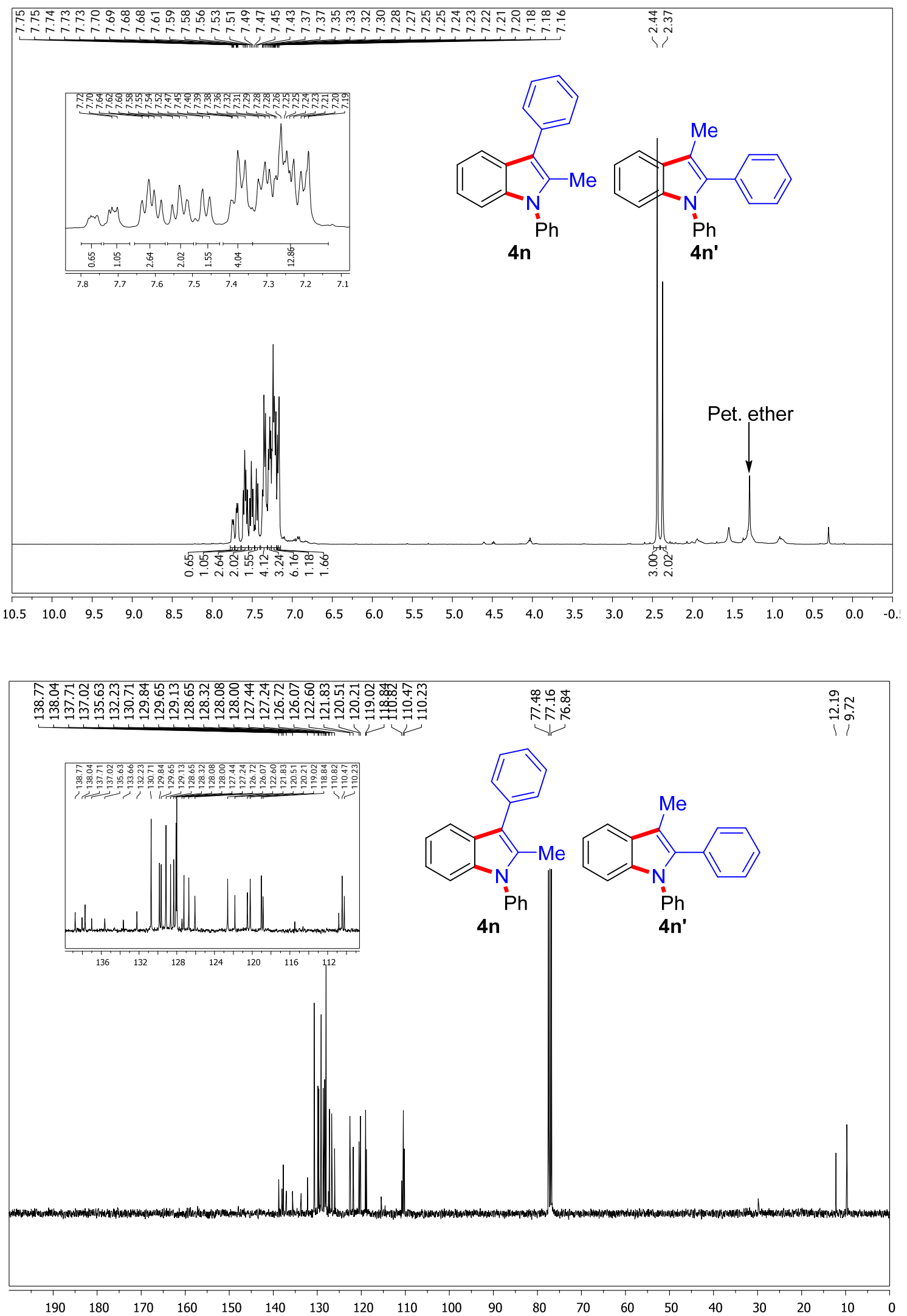
1-(2,5-Dimethylphenyl)-4,7-dimethyl-2,3-diphenyl-1H-indole (4o)
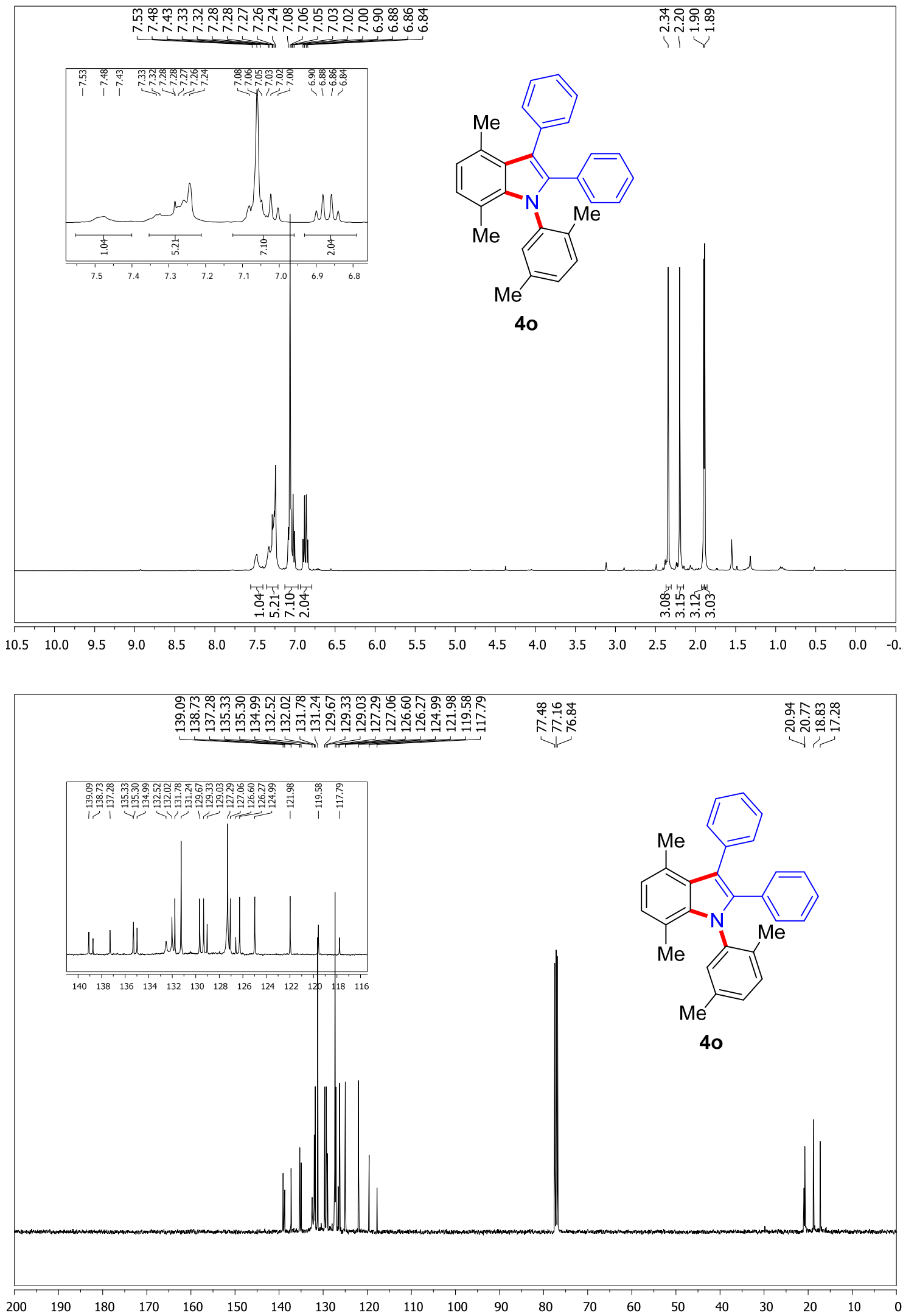


\section{1,2,3-Triphenyl-1H-benzo[f]indole (4p)}
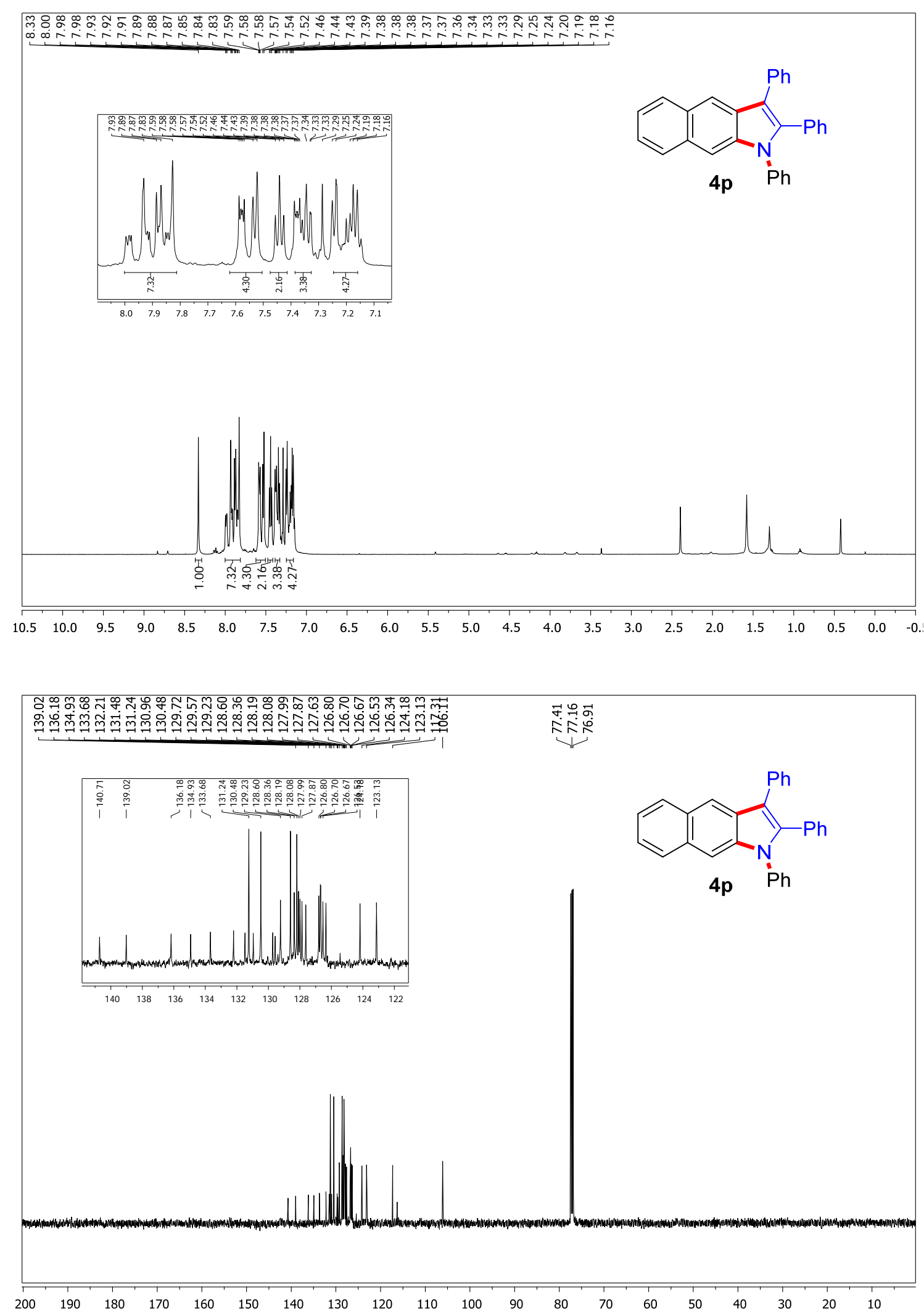
5-(Benzo[d][1,3]dioxol-4-yl)-6,7-diphenyl-5H-[1,3]dioxolo[4,5-f]indole (4q)
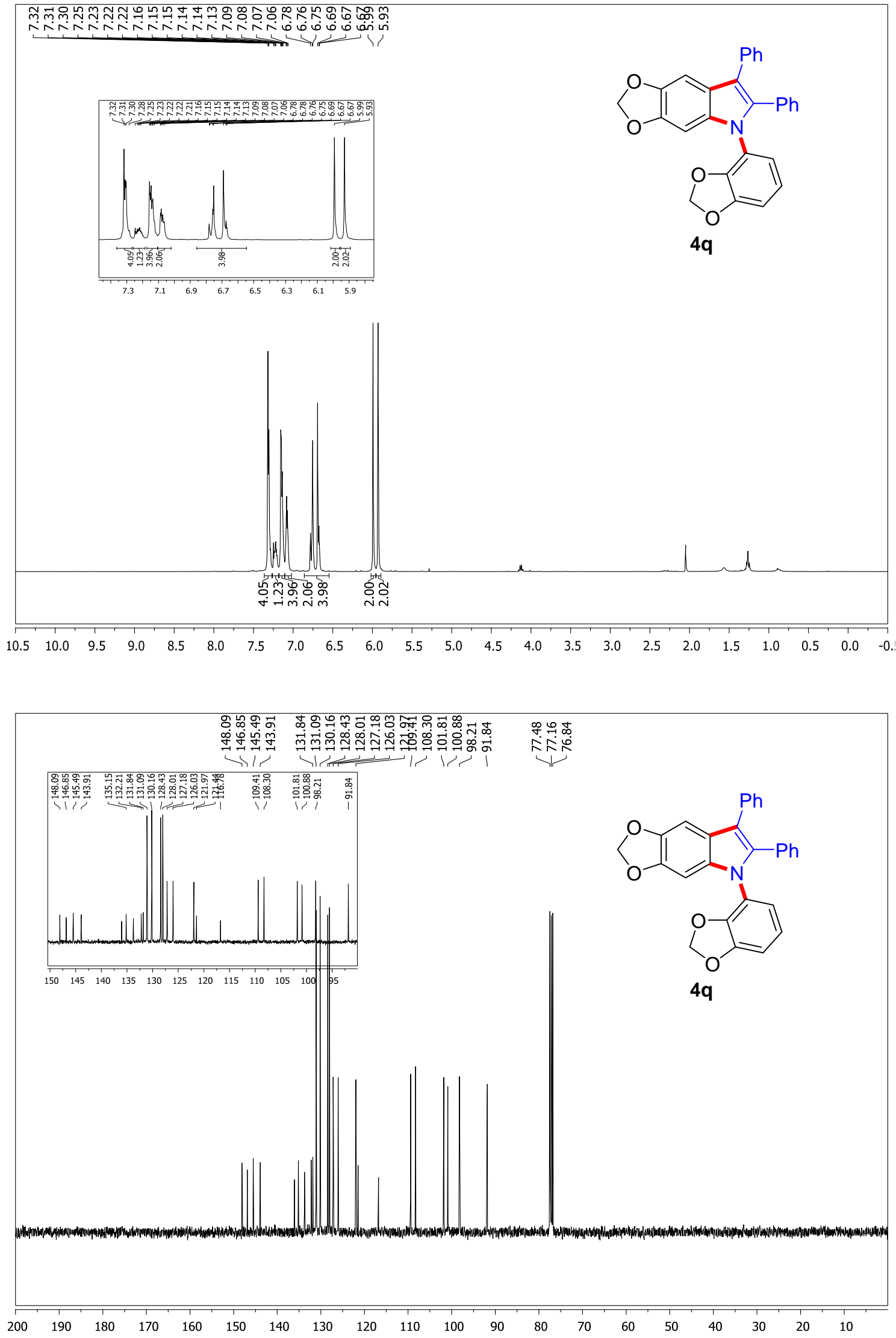


\section{5. ${ }^{1} \mathrm{H}$ and ${ }^{13} \mathrm{C}$ NMR Spectra of indolo[2,1-a]isoquinoline derivative}

\section{5,6,12-Triphenylindolo[2,1-a]isoquinoline (6a)}

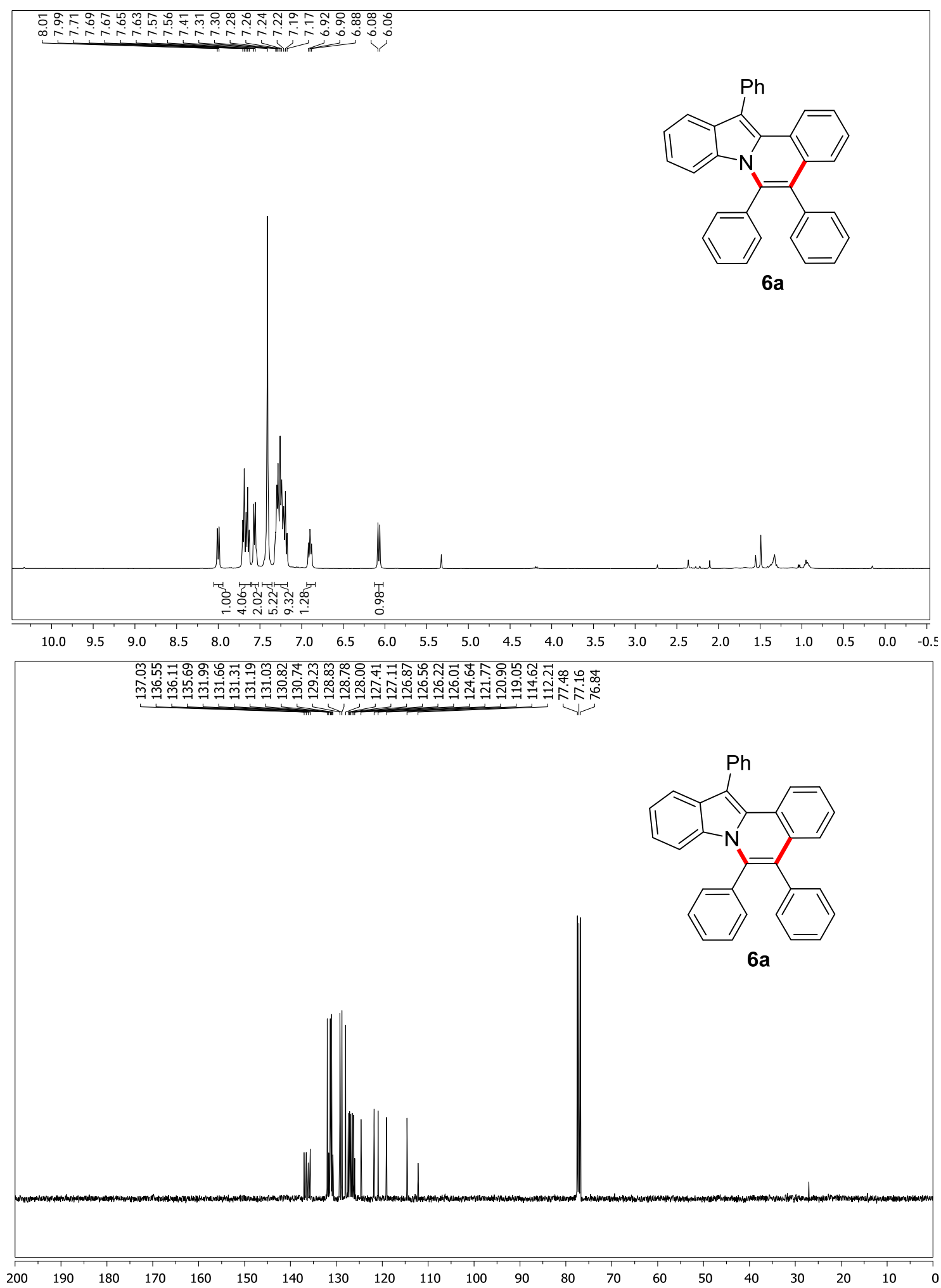

